\title{
Asymptotic Properties of Solutions to the Cauchy Problem for Degenerate Parabolic Equations with Inhomogeneous Density on Manifolds
}

\author{
Daniele Andreucciø and Anatoli F. Tedeev
}

\begin{abstract}
We consider the Cauchy problem for doubly nonlinear degenerate parabolic equations with inhomogeneous density on noncompact Riemannian manifolds. We give a qualitative classification of the behavior of the solutions of the problem depending on the behavior of the density function at infinity and the geometry of the manifold, which is described in terms of its isoperimetric function. We establish for the solutions properties as: stabilization of the solution to zero for large times, finite speed of propagation, universal bounds of the solution, blow up of the interface. Each one of these behaviors of course takes place in a suitable range of parameters, whose definition involves a universal geometrical characteristic function, depending both on the geometry of the manifold and on the asymptotics of the density at infinity.
\end{abstract}

Mathematics Subject Classification. 35K55, 35K65, 35B40.

Keywords. Doubly degenerate parabolic equation, Noncompact Riemannian manifold, Inhomogeneous density, Interface blow up, Optimal decay estimates.

\section{Introduction}

We consider the Cauchy problem

$$
\begin{aligned}
\rho(x) u_{t}-\Delta_{p, m}(u) & =0, \quad x \in M, t>0, \\
u(x, 0) & =u_{0}(x), \quad x \in M .
\end{aligned}
$$

We consider in fact only non-negative solutions to (1.1) and (1.2). Here $M$ is a complete Riemannian manifold of topological dimension $N$, with infinite volume. In

D. Andreucci is member of the Gruppo Nazionale per la Fisica Matematica (GNFM) of the Istituto Nazionale di Alta Matematica (INdAM). A. F. Tedeev was supported by Sapienza Grant C26V17KBT3. 
local coordinates $x^{i}$, we denote

$$
\Delta_{p, m}(u)=\frac{1}{\sqrt{\operatorname{det}\left(g_{i j}\right)}} \sum_{i, j=1}^{N} \frac{\partial}{\partial x^{i}}\left(\sqrt{\operatorname{det}\left(g_{i j}\right)} g^{i j} u^{m-1}|\nabla u|^{p-2} \frac{\partial u}{\partial x^{j}}\right),
$$

where $\left(g_{i j}\right)$ denotes the Riemannian metric, $\left(g^{i j}\right)=\left(g_{i j}\right)^{-1}, \nabla u$ is the gradient with respect to $\left(g_{i j}\right)$, and

$$
|\nabla u|^{2}=\sum_{i, j=1}^{N} g^{i j} \frac{\partial u}{\partial x^{j}} \frac{\partial u}{\partial x^{i}} .
$$

We always assume that $1<p<N$, and that either

$$
p+m-3>0,
$$

or

$$
p+m-3<0, \quad m>0 .
$$

In this paper we follow an approach ultimately based on the classical DeGiorgi estimates $[13,26]$; a new technical tool is the weighted Faber-Krahn type inequality of Lemmas 2.10 and 2.11. This inequality takes into account the asymptotic behaviors both of the density function $\rho$ and of the volume growth of the manifold at infinity. We use the isoperimetrical properties of the manifold, which also allow us to prove new embedding results which we think are of independent interest.

We establish in the slow diffusion case (1.3), and also in the fast diffusion case (1.4) under additional assumptions, the decay rate for large times of nonnegative solutions, for initial data of finite mass. In the degenerate case we also estimate the finite speed of propagation for the support of solutions with a bounded support. These results apply in a subcritical case where, roughly speaking, the density function decays not too fast at infinity. Where we have explicit solutions, that is in the Euclidean case, our estimates reduce to the known optimal ones.

Still in the slow diffusion case, but when the density function decays fast enough, we investigate the behavior of the solutions for large $t$; we obtain under different assumptions a universal bound for solutions and a result of interface blow up. The universal bound is the same as in the Euclidean case, as expected; see also Remark 1.1.

The various cases recalled above are discriminated in terms of the behavior of a universal function involving the density and the volume growth of the manifold (see Remark 1.4).

The interest of this problem appeared first in the case $M=\boldsymbol{R}^{3}$ with the Euclidean metric, where $[25,38]$ obtained the first surprising results, in symmetric cases, on the qualitative properties of solutions to the porous media equation with inhomogeneous density. The interface blow up in the Euclidean setting was first shown in [23]; [42] extended it to a wide class of doubly degenerate parabolic equations.

Remark 1.1. (The Euclidean case $M=\boldsymbol{R}^{N}$ ) Let us here explicitly recall the behavior of solutions in the Euclidean case, when $\rho(x)=(1+|x|)^{-\alpha}$ in (1.1), for $x \in M=\boldsymbol{R}^{N}$, for a given $0<\alpha<N$, in the degenerate case (1.3). Such a behavior 
strongly depends on the interplay between the nonlinearities appearing in the equation, at least from a two-fold point of view, that is both as far as the sup bounds for solutions are concerned, and then also if we consider the property of finite speed of propagation, see [42] for the following results; see also [15].

Concerning the first issue, the limiting threshold is $\alpha=p$ : in the range $\alpha<p$ the sup estimates for solutions depend on the initial data in the spirit of what is known for the homogeneous doubly nonlinear equation. More explicitly,

$$
\|u(t)\|_{\infty} \leq \gamma t^{-\frac{N-\alpha}{(N-\alpha)(m+p-3)+p-\alpha}}\left\|\rho u_{0}\right\|_{1}^{\frac{p-\alpha}{(N-\alpha)(m+p-3)+p-\alpha}}, \quad t>0 .
$$

Instead in the range $\alpha>p$ one can prove a universal bound: the initial mass disappears from the estimate; more exactly for a $\gamma$ independent of the solution we have

$$
\|u(t)\|_{\infty} \leq \gamma t^{-\frac{1}{m+p-3}}, \quad t>0 .
$$

Concerning the second issue of finite speed of propagation, which is connected to conservation of mass, a second explicit threshold appears, given by

$$
\alpha_{*}=\frac{N(m+p-3)+p}{m+p-2} \in(p, N) .
$$

Clearly, we assume that the initial data has compact support in, say, $B_{R_{0}}(0)$. Then in the subcritical range $\alpha<\alpha_{*}$ the property of finite speed of propagation is preserved for all times, i.e., the support of the solution at time $t$ is contained in the ball centered at the origin with radius $R(t)$ where

$$
R(t)=4 R_{0}+\gamma\left\|\rho u_{0}\right\|_{1}^{\frac{m+p-3}{(N-\alpha)(m+p-3)+p-\alpha}} t^{\frac{1}{(N-\alpha)(m+p-3)+p-\alpha}}, \quad t>0
$$

the exponent of $t$ in (1.8) is positive if and only if $\alpha<\alpha_{*}$. As a result, also the property of conservation of mass is valid for all times.

In the supercritical range $\alpha>\alpha_{*}$ the evolution of the support is quite different: finite speed of propagation (that is boundedness of support) and conservation of mass can not hold true for all $t>0$.

In Sects. 1.1.1 and 1.1.2 below we present examples which can be compared with the Euclidean case. Before describing the results of this paper, we recall that parabolic problems in a Euclidean metric with inhomogeneous density were studied in $[27,28]$ (blow up phenomena); $[24,36]$ (asymptotic expansion of the solution of the porous media equation); $[22,31]$ (critical case). We quote for results related to ours $[19,21,32]$ for the porous media equation and [14] for anisotropic operators. Still on the subject of porous media like equations on Riemannian manifolds, besides the seminal papers $[33,35]$ on the properties of the support of solutions, we also quote $[20,44]$.

The main goal of the present paper is to investigate the behavior of solutions around the threshold discriminating between the cases described above for the sup estimates, in terms of the density function $\rho$, the nonlinearities in the equation, and of course the Riemannian geometry of $M$.

Our results on the subcritical $L^{\infty}$ bound in fact apply when we, clearly, are in the subcritical case, but not too far from the threshold. In the Euclidean example just 
discussed, i.e., $\alpha$ should be not too small, which is not restrictive in the light of the purpose of this paper. On the other hand, we provide a unified approach to both the degenerate (1.3) and the singular (1.4) cases. The case where we are far below the threshold calls for a different approach; this will be the subject of a forthcoming paper.

Such a requirement of closeness to the threshold is not needed by the other results. See also [1] for the Euclidean case; we borrow the energetic setting of $[4,6-8]$; see also [41].

\subsection{Assumptions}

In what follows $d(x)$ denotes the geodesic distance of $x$ from a fixed point $x_{0} \in M$, $|U|$ is the Riemannian volume of $U \subset M$, and $|\partial U|_{N-1}$ the corresponding area of its boundary.

An important role is played by the function

$$
V(R)=\left|B_{R}\right|, \quad B_{R}=\{x \in M \mid d(x) \leq R\} .
$$

On the geometry of the manifold $M$ we need the following requirements, of isoperimetrical character. We assume that for all bounded and Lipschitz domains $U \subset M$

$$
|\partial U|_{N-1} \geq h(|U|), \quad s \mapsto \omega(s)=\frac{s^{\frac{N-1}{N}}}{h(s)} \text { is nondecreasing in }(0,+\infty) .
$$

Here $h:[0,+\infty) \rightarrow[0,+\infty)$ is a continuous nondecreasing function, with $h(0)=0$.

Remark 1.2. The connection between isoperimetrical inequalities and embedding theorems in $\boldsymbol{R}^{N}$ is well known, see [29]; for a discussion of the connection of embeddings in Riemannian manifolds with Sobolev and Gagliardo-Nirenberg inequalities in the Euclidean space we refer to [9, Chapter 2]. See the classical [43] for the connection between the heat semigroup and isoperimetric inequalities.

Also inequalities of Faber-Krahn type have long been known to be useful in investigating the behavior of solutions to parabolic equations in manifolds, see e.g., [18] for connections to the heat kernel bounds, and [7] for the nonlinear case.

Roughly speaking, if the isoperimetric profile satisfies $h(v) \simeq c_{1} v^{a}$ for large $v>0$ and a fixed $c_{1}>0$, then we must require $a>(p-1) / p$, which amounts essentially to the nonparabolicity of the manifold (see (1.22)) and leads to $p<N$ in the Euclidean case where $a=(N-1) / N$. In particular, in the linear case $p=2$ we must have $N \geq 3$.

1.1.1. Example: Symmetric Manifolds. As a first interesting example let us consider the case of a model Riemannian manifold (see e.g., [17]) with metric $\mathrm{d} r^{2}+f(r)^{2} \mathrm{~d} \xi^{2}$, $r \geq 0, \xi \in S^{N-1}$. We have

$$
V(R)=\omega_{N} \int_{0}^{R} f(s)^{N-1} \mathrm{~d} s, \quad \omega_{N}=\left|S^{N-1}\right|_{N-1} .
$$

We define the continuous functions $f, \rho$ for $\tau \geq 0$ as in

$$
f(\tau)=\left\{\begin{array}{ll}
C(A) \tau, & \tau \leq A, \\
\tau^{\beta}(\ln \tau)^{\nu}, & \tau>A ;
\end{array} \quad \rho(\tau)= \begin{cases}B^{-\alpha}(\ln B)^{\mu}, & \tau \leq B, \\
\tau^{-\alpha}(\ln \tau)^{\mu}, & \tau>B,\end{cases}\right.
$$


where $B \geq A \geq e$ are suitably chosen. Here $(p-1) /(N-1)<\beta<1$ and $\mu, \nu \in \boldsymbol{R}$, $\alpha>0$. In this case one can take

$$
h(s)=c_{0} f\left(V^{(-1)}(s)\right)^{N-1},
$$

where $c_{0}>0$ is a suitable constant. Thus for suitable $c_{1}, c_{2}>0$

$$
h(s) \simeq c_{1} s^{\frac{N-1}{N}}, s \rightarrow 0 ; \quad h(s) \simeq c_{2} s^{\frac{\beta(N-1)}{\beta(N-1)+1}}(\ln s)^{\frac{\nu(N-1)}{\beta(N-1)+1}}, s \rightarrow+\infty .
$$

By means of lengthy but straightforward calculations one can check that the assumptions appearing in the statements of Sect. 1.2 below are satisfied, thereby implying the following results.

We prove the analog of the subcritical sup estimate (1.5) (see Theorem 1.7) in the two cases:

(i) $p+m-3<0, m>0$ : we have to assume

$$
\begin{aligned}
& N(p+m-3)+p>0, \\
& \alpha<\frac{(\beta(N-1)+1)(p+m-3)+p}{p+m-2}=: \alpha_{s}^{*}, \\
& p>\alpha>\frac{N-1}{N}(1-\beta) p^{*}, \quad p^{*}=\frac{N p}{N-p} .
\end{aligned}
$$

(ii) $p+m-3>0$ : in this case (1.11) is automatically satisfied. We assume (1.12), (1.13).

In these cases we have for large $t>0$

$$
\|u(t)\|_{\infty} \leq \gamma\left(\left\|\rho u_{0}\right\|_{1}\right) t^{-\delta_{1}}(\ln t)^{\delta_{2}}
$$

where

$$
\begin{aligned}
& \lambda=p-\alpha+(1+\beta(N-1)-\alpha)(p+m-3), \\
& \sigma=\nu(N-1)(p+m-3)+\mu(p+m-2), \\
& \delta_{1}=\frac{\beta(N-1)+1-\alpha}{\lambda}, \quad \delta_{2}=\sigma \delta_{1}-\mu-\nu(N-1) .
\end{aligned}
$$

Here $\lambda>0$ owing to our assumptions.

We can prove for large times (see Theorem 1.8) the following estimate of finite speed of propagation (compare with (1.8)), provided $p+m-3>0$ and (1.12) is satisfied:

$$
R(t) \leq 4 R_{0}+\gamma\left(\left\|\rho u_{0}\right\|_{1}\right) t^{\frac{1}{\lambda}}(\ln t)^{-\frac{\sigma}{\lambda}} .
$$

Concerning the universal bound result in (1.6), if in this example we assume $\alpha>p$, $p+m-3>0$ the same estimate (1.6) holds true (with a different constant $\gamma>0$ ); see Theorem 1.9.

Finally, the interface blow up phenomenon takes place if $p+m-3>0$ and

$$
\alpha>\alpha_{s}^{*},
$$

note that $\alpha_{s}^{*}>p$; see Theorem 1.10 and observe that (1.18) guarantees (1.40). For the reader's convenience and with reference to the notation employed below, we mention that in this example the function $\psi$ (see Remark 1.4) takes the form

$$
\psi(s)=s^{\lambda}(\ln s)^{\sigma}(1+\mathrm{o}(1)), \quad s \rightarrow+\infty,
$$


so that

$$
\psi^{(-1)}(v)=\lambda^{\frac{\sigma}{\lambda}} v^{\frac{1}{\lambda}}(\ln v)^{-\frac{\sigma}{\lambda}}(1+\mathrm{o}(1)), \quad v \rightarrow+\infty .
$$

We also note that if we take formally above $\beta=1, \mu=\nu=0$, we recover the results known in the setting of the Euclidean space [42]. Furthermore, the example is still admissible, under the same assumptions, if $f$ and $\rho$ are modified by multiplying them by a factor $1+H(\tau)$ where $H$ is a sufficiently regular and fast decaying function as $\tau \rightarrow+\infty$.

1.1.2. Example: Manifolds with Bounded Geometry. A complete Riemannian manifold $M$ is said to have bounded geometry if its Ricci curvature is bounded from below by a negative constant, and its injectivity radius is bounded from below by a positive constant; see [11, Theorem V.2.6] for the following result (which we state in our notation): Let $M$ have bounded geometry and $\rho>0, r>1$; if (and only if) there exists $c>0$ such that

$$
|\partial U|_{N-1} \geq c|U|^{1-\frac{1}{r}}
$$

for all smooth open submanifolds $U$ with compact closure containing a closed disk of radius $\rho$, then we may take

$$
h(s)=\theta \min \left(s^{1-\frac{1}{N}}, s^{1-\frac{1}{r}}\right),
$$

for a suitable $\theta>0$. In our case we have to assume that $p<r \leq N$.

We single out from the previous class of examples the subcase of manifolds $M_{d} \times$ $\boldsymbol{R}^{k}$, where $M_{d}$ is a compact Riemannian manifold of dimension $d \geq 1$ (see [11, Examples V.2.3, V.3.6]). In this case we have in (1.17) that $N=d+k, r=k$. Such product manifolds have been the subject of much interest in the literature (see $[37,39])$. In this case the results outlined in the previous example still hold when in them we formally let $\beta=(k-1) /(N-1), \nu=0$; the density $\rho$ is taken as in the previous example. More explicitly, the assumptions we need in the case $p+m-3<0, m>0$ are (1.11) (with $N=d+k)$ and

$$
\begin{aligned}
& \alpha<\frac{k(p+m-3)+p}{p+m-2}=: \alpha_{b}^{*}, \\
& p>\alpha>\frac{d}{d+k} p^{*}
\end{aligned}
$$

in the case $p+m-3>0$ we have only to assume (1.18) and (1.19). We also compute here

$$
\begin{aligned}
& \lambda=p-\alpha+(k-\alpha)(p+m-3), \quad \sigma=\mu(p+m-2), \\
& \delta_{1}=\frac{k-\alpha}{\lambda}, \quad \delta_{2}=\sigma \delta_{1}-\mu .
\end{aligned}
$$

Then (1.14) holds true with $\delta_{1}, \delta_{2}$ as above.

Let us assume next $p+m-3>0$ : then the finite speed of propagation estimate (1.15) still holds, under assumption (1.18); if instead $\alpha>p$, the universal bound (1.6) is in force, while in the case $\alpha>\alpha_{b}^{*}$ we have interface blow up. 
We list here all the assumptions required in the following; all of them are needed for the subcritical sup estimate, while the other results employ only a subset of such hypotheses.

1.1.3. Volume Growth Assumptions. We require the growth conditions

$$
\operatorname{ch}(V(R)) \leq \frac{\mathrm{d} V}{\mathrm{~d} R}(R), \quad R>0,
$$

and its counterpart

$$
\frac{\mathrm{d} V}{\mathrm{~d} R}(R) \leq c^{-1} h(V(R)), \quad R>0,
$$

for a given $0<c<1$.

The following condition of non-parabolicity of the manifold is needed to prove global embedding results:

$$
\int_{0}^{k} \frac{\mathrm{d} t}{V^{(-1)}(t)^{p}} \leq c^{-1} \frac{k}{V^{(-1)}(k)^{p}}, \quad k>0 .
$$

In some cases we need that $R^{N} / V(R)$ is nondecreasing, which is implied by the assumption

$$
\frac{\mathrm{d} V}{\mathrm{~d} R}(R) \leq N \frac{V(R)}{R}
$$

In addition we require

$$
\frac{V(R)}{R} \leq c^{-1} h(V(R)), \quad R>0 .
$$

1.1.4. Density Decay Assumptions. In order to get the subcritical sup estimate, the density function $\rho$ is required to satisfy:

$$
\begin{aligned}
& s \mapsto \rho(s) s^{\alpha_{1}} \text { is nonincreasing for } s \in(1,+\infty) ; \\
& s \mapsto \rho(s) s^{\alpha_{2}} \text { is nondecreasing for } s \in(1,+\infty),
\end{aligned}
$$

where $0<\alpha_{1}<\alpha_{2}<p$ are given constants.

Note that (1.22) and (1.26) imply that the function $V_{\rho}(R)=\rho(R) V(R)$ is bounded from above and below by constant multiples of the same increasing function (see Lemma 2.4 below); however we need state the more precise assumption

$$
V_{\rho}(R)=\rho(R) V(R) \text { is increasing for } R \in(0,+\infty) .
$$

We denote the inverse function of $V_{\rho}$ by $R_{\rho}$. This hypothesis will be assumed implicitly throughout.

Finally, as we remarked above, we need to be not too far from the threshold, in Theorem 1.7; exactly, this means that, on setting $p^{*}=N p /(N-p)$,

$$
s \mapsto \rho(s) \omega(V(s))^{p^{*}}, \text { is nonincreasing in } s>s_{0},
$$

for a suitably chosen $s_{0}>0$. 
Remark 1.3. One can see easily that assumption (1.22) is a consequence of the following alternative assumption: $R \mapsto V(R) / R^{q}$ is nondecreasing for $R>0$ for some $q>p$.

Assumption (1.22) is not merely a technical device; if it fails we do not expect decay of solutions for large times, see [25].

Remark 1.4. Consider the functions

$$
\begin{aligned}
& \psi(R)=V_{\rho}(R)^{p+m-3} \rho(R) R^{p}, \quad R>0 ; \\
& b_{1}(s)=\psi\left(R_{\rho}(s)\right)=s^{p+m-3} \rho\left(R_{\rho}(s)\right) R_{\rho}(s)^{p}, \quad s>0 .
\end{aligned}
$$

Owing to our assumptions, $b_{1}$ is increasing if and only if $\psi$ is. In turn, this is automatically satisfied in the degenerate case (1.3), at least if (1.26) is assumed, but it is not necessarily valid in the singular case (1.4). However, when $\psi$ is increasing we denote its inverse by $\tilde{Z}$; the latter function provides in some cases an estimate of the finite speed of propagation of the support of a solution.

The definition of weak solution to (1.1) and (1.2) is in fact standard; the problem is given the integral formulation

$$
\int_{0}^{+\infty} \int_{M}\left\{-u \rho \zeta_{t}+u^{m-1}|\nabla u|^{p-2} \nabla u \nabla \zeta\right\} \mathrm{d} \mu \mathrm{d} t=\int_{M} u_{0} \rho \zeta(x, 0) \mathrm{d} \mu
$$

for all $\zeta \in C^{1}(M \times[0,+\infty))$, with bounded support. In general, the existence can be proved following the methods of $[9,10]$ in the setting of energy solutions, i.e., assuming $\sqrt{\rho} u_{0} \in L^{2}(M)$. The solution obtained satisfies $u \in L_{\text {loc }}^{\infty}(M \times(0,+\infty))$, $u \in C\left((0, T) ; L^{2}(M)\right), u^{m-1}|\nabla u|^{p} \in L^{1}(M \times(0, T))$ for all $T<+\infty$. In the subcritical cases where the assumptions of Theorem 1.7 are in force, in fact we can prove existence even for initial data which are Radon measures, such that $\rho u_{0}$ has finite mass in $M$. This follows from the estimate in Theorem 1.7 and from a standard approximation procedure via a sequence of solutions to initial-value boundary problems, with vanishing Dirichlet data, in a sequence of invading compact domains. In the latter case however the solution satisfies $u \in L_{\mathrm{loc}}^{\infty}(M \times(0,+\infty))$, $u \in C\left((0, T) ; L_{\text {loc }}^{2}(M)\right), u^{m-1}|\nabla u|^{p} \in L_{\text {loc }}^{1}(M \times(0, T))$ for all $T<+\infty$. In addition, the proof requires an estimate of the $L^{1}$ norm of $u^{m-1}|\nabla u|^{p-1}$ up to time $t=0$ (sometimes called an entropy estimate); this bound can be achieved following $[3,5]$, and again using Theorem 1.7.

The uniqueness of solutions for problems of the kind we consider is well known to be a difficult problem in general; see e.g., the seminal paper [16], and more recently [34].

Thus in the following theorems, we refer to a solution $u$ constructed by approximation as shown above. Also, in the proofs for simplicity we work sometimes with a strong almost everywhere formulation of the differential equation, to avoid the by now standard regularization arguments. We denote by $\gamma, \gamma_{0}, \ldots$, constants (varying from line to line) depending only on the parameters of the problem.

Remark 1.5. Since we can limit the $L^{1}(M)$ norm of each such approximation only in terms of the initial mass, passing to the limit we infer for the solution referred to 
just above

$$
\|u(t) \rho\|_{L^{1}(M)} \leq \gamma\left\|u_{0} \rho\right\|_{L^{1}(M)}, \quad 0<t<+\infty .
$$

Here $\gamma$ depends on $M$ and $\rho$, but not on $u$. Notice that this bound follows without assuming finite speed of propagation. However, it is easy to prove that if the support of the solution is bounded over $[0, T]$, mass is conserved exactly, that is

$$
\|u(t) \rho\|_{L^{1}(M)}=\left\|u_{0} \rho\right\|_{L^{1}(M)}, \quad 0<t<T .
$$

Remark 1.6. It follows without difficulty from our arguments that the radial character and the assumptions on $\rho$ can be replaced by analogous statements on a radial function $\tilde{\rho}$ such that

$$
c \tilde{\rho}(x) \leq \rho(x) \leq c^{-1} \tilde{\rho}(x), \quad x \in M,
$$

for a given $0<c<1$.

\subsection{Main Results}

We begin with the subcritical sup estimate.

Theorem 1.7. Assume (1.9)-(1.28). We also assume one of the following: (i) $p+$ $m-3<0, m>0, \psi$ as in (1.29) is increasing with $\psi(0+)=0, \psi(R) \rightarrow+\infty$ as $R \rightarrow+\infty$, and

$$
\left(N-\alpha_{1}\right)(p+m-3)+p-\alpha_{2}>0
$$

(ii) $p+m-3>0$ (in this case the other conditions in (i) are automatically satisfied). Let $\rho u_{0} \in L^{1}(M)$. Then for $\tilde{Z}=\psi^{(-1)}$,

$$
\|u(t)\|_{\infty} \leq \frac{\left\|\rho u_{0}\right\|_{1}}{V_{\rho}\left(\tilde{Z}\left(\gamma_{0} t\left\|\rho u_{0}\right\|_{1}^{p+m-3}\right)\right)}, \quad t>0,
$$

for a constant $\gamma_{0}>0$ independent of $u$.

Next we deal with the finite speed of propagation.

Theorem 1.8. Assume that $\operatorname{supp} u_{0} \subset B_{R_{0}}$ and that we are in the degenerate case (1.3), with $\psi$ increasing, $\psi(0+)=0$. Assume further (1.9) and that for a suitable $C>0$

$$
\rho(r) \leq C \rho(2 r), \quad r \geq 0 .
$$

Let $\rho u_{0} \in L^{1}(M)$. Then for all $t>0, \operatorname{supp} u(t) \subset B_{R}$ if $R$ satisfies

$$
R=4 R_{0}+\tilde{Z}\left(\gamma t\left\|\rho u_{0}\right\|_{1}^{p+m-3}\right),
$$

for a constant $\gamma>0$ independent of $u$.

The reason why we can avoid in Theorem 1.8 several of the global assumptions of Theorem 1.7 is that in the proof we work in bounded sets, shaped like annuli. Thus we don't need to apply a weighted Sobolev inequality, which dispenses us from assuming non-parabolicity as in (1.22). By the same token, we need only a standard doubling property for $\rho$.

In the supercritical case (1.38) we can prove the following universal, or absolute, sup bound for the solution, which is in fact in its functional form independent of the initial data and of the geometry of $M$. 
Theorem 1.9. Assume that (1.3), (1.9)-(1.24) hold true, and that for a suitable $c>$ 0

$$
\rho(\tau) \leq c^{-1} \tau^{-\alpha}, \quad \tau>1
$$

for some $\alpha>p$.

Let $\rho u_{0} \in L^{1}(M), \sqrt{\rho} u_{0} \in L^{2}(M)$. Then

$$
\|u(t)\|_{\infty} \leq \gamma t^{-\frac{1}{p+m-3}}, \quad t>0
$$

where $\gamma$ does not depend on $u$.

Finally, if the second threshold is exceeded, which we essentially assume in (1.40), the finite speed of propagation property fails.

Theorem 1.10. Assume (1.3), (1.9), (1.22), (1.23), (1.24). Let $\rho u_{0} \in L^{1}(M), \sqrt{\rho} u_{0} \in$ $L^{2}(M)$ and $u_{0}$ with bounded support. Assume that

$$
\int_{1}^{+\infty}\left(\tau^{p} \rho(\tau)\right)^{r} \psi(\tau)^{\frac{1}{p+m-3}} \frac{\mathrm{d} \tau}{\tau}<+\infty,
$$

for $r \in\left(-r_{0}, r_{0}\right)$ for some $r_{0}>0$.

Then the boundedness of the support of $u(t)$ fails over $(0, \bar{t})$ for a sufficiently large $\bar{t}>0$.

\subsection{Plan of the Paper}

We prove in Sect. 2 several auxiliary inequalities. In Sect. 3 we prove Theorem 1.7 in the degenerate case, while the proof in the singular case, being a minor variant of the previous one, is dealt with in the short Sect. 4. The finite speed of propagation property is proved in Sect. 5. The supercritical universal sup bound is proved in Sect. 6, while finally the interface blow up is treated in Sect. 7.

\section{Auxiliary Results}

We begin by stating the following trivial result.

Lemma 2.1. Let $f \in C([0,+\infty))$ be a nonnegative function such that for given $r_{0}$, $c_{0}, c_{1}, \beta>0, f$ is nondecreasing in $\left(r_{0},+\infty\right)$ and satisfies

$$
c_{0} s^{\beta} \leq f(s) \leq c_{1} s^{\beta}, \quad 0 \leq s \leq r_{0} .
$$

Then for all $s>r>0$

$$
f(r) \leq c_{1} c_{0}^{-1} f(s)
$$

A first consequence of Lemma 2.1 applied to $f(s)=\rho(s) s^{\alpha_{2}}$, and of (1.26), is the following one: for all $s>r>0$

$$
\rho(r) r^{\alpha_{2}} \leq C \rho(s) s^{\alpha_{2}}
$$

where $C=\rho(0) / \rho(1) \geq 1$. 
Lemma 2.2. Assume (1.22); then for the same constant c as in (1.22)

$$
V(s) \geq c\left(\frac{s}{r}\right)^{p} V(r), \quad s>r>0 .
$$

Assume further (1.26); then a constant $\tilde{c}>0$ exists such that

$$
V_{\rho}(s) \geq \tilde{c}\left(\frac{s}{r}\right)^{p-\alpha_{2}} V_{\rho}(r), \quad s>r>0 .
$$

As a consequence

$$
R_{\rho}(a) \leq \tilde{c}^{-\frac{1}{p-\alpha_{2}}}\left(\frac{a}{b}\right)^{\frac{1}{p-\alpha_{2}}} R_{\rho}(b), \quad a>b>0 .
$$

Proof. The inequality (2.4) follows from (1.22), since on taking there $k=V(s)$ we get

$$
c^{-1} \frac{V(s)}{s^{p}} \geq \int_{0}^{V(s)} \frac{\mathrm{d} t}{V^{(-1)}(t)^{p}} \geq \int_{0}^{V(r)} \frac{\mathrm{d} t}{V^{(-1)}(t)^{p}} \geq \frac{V(r)}{r^{p}},
$$

when we also exploit the fact that the integrand is decreasing.

Next we compute appealing to (2.3) and to (2.4)

$$
V_{\rho}(s)=V(s) \rho(s) s^{\alpha_{2}} s^{-\alpha_{2}} \geq c\left(\frac{s}{r}\right)^{p} V(r) C^{-1} \rho(r) r^{\alpha_{2}} s^{-\alpha_{2}},
$$

whence (2.5). Finally (2.6) is a simple consequence of (2.5).

Lemma 2.3. Under the assumptions (1.9), (1.20), (1.21) we have

$$
\begin{array}{ll}
\gamma^{-1} \lambda V(R) \leq V(\lambda R) \leq \gamma \lambda^{N} V(R), & \lambda \geq 1, \\
\gamma^{-1} \lambda^{N} V(R) \leq V(\lambda R) \leq \gamma \lambda V(R), & 0<\lambda \leq 1 .
\end{array}
$$

Proof. Define $F(v)=v / h(v)$, for $v>0$. Owing to our assumption (1.9) we have for $k \geq 1$

$$
F(k v)=\frac{k v}{h(k v)}=\frac{k v}{(k v)^{\frac{N-1}{N}}} \frac{(k v)^{\frac{N-1}{N}}}{h(k v)} \geq k^{\frac{1}{N}} F(v) .
$$

Similarly for $0<k \leq 1$ and $v>0$

$$
F(k v) \leq k^{\frac{1}{N}} F(v) .
$$

It follows that $F$ is increasing and

$$
F(v) \rightarrow 0, \quad v \rightarrow 0+, \quad F(v) \rightarrow+\infty, \quad v \rightarrow+\infty .
$$

In addition, from the monotonicity of $h$ we get

$$
F(\lambda v) \leq \lambda F(v) \quad \lambda \geq 1 ; \quad F(\lambda v) \geq \lambda F(v), \quad 0<\lambda \leq 1 .
$$

Then for given $\lambda, R>0$ we let $v=F^{(-1)}(R), k=\lambda^{N}$ and infer from (2.11)-(2.13),

$$
\begin{array}{ll}
\lambda F^{(-1)}(R) \leq F^{(-1)}(\lambda R) \leq \lambda^{N} F^{(-1)}(R), & \lambda \geq 1 ; \\
\lambda^{N} F^{(-1)}(R) \leq F^{(-1)}(\lambda R) \leq \lambda F^{(-1)}(R), & 0<\lambda \leq 1 .
\end{array}
$$


Next we invoke assumptions (1.20), (1.21) to infer

$$
\begin{aligned}
c^{-1} R & =c^{-1} \int_{0}^{V(R)}\left(\frac{\mathrm{d} V}{\mathrm{~d} R}(s)\right)^{-1} \mathrm{~d} s \geq \int_{0}^{V(R)} \frac{\mathrm{d} s}{h(s)} \\
& \geq c \int_{0}^{V(R)}\left(\frac{\mathrm{d} V}{\mathrm{~d} R}(s)\right)^{-1} \mathrm{~d} s=c R .
\end{aligned}
$$

However, again from (1.9),

$$
\int_{0}^{V(R)} \frac{\mathrm{d} s}{h(s)}=\int_{0}^{V(R)} s^{-\frac{N-1}{N}} \frac{s^{\frac{N-1}{N}}}{h(s)} \mathrm{d} s \leq N \frac{V(R)}{h(V(R))}=N F(V(R)),
$$

while invoking again the monotonicity of $h$

$$
\begin{gathered}
\int_{0}^{V(R)} \frac{\mathrm{d} s}{h(s)} \geq \int_{\frac{V(R)}{2}}^{V(R)} \frac{\mathrm{d} s}{h(s)}=\int_{\frac{V(R)}{2}}^{V(R)} s^{-\frac{N-1}{N}} \frac{s^{\frac{N-1}{N}}}{h(s)} \mathrm{d} s \\
\geq \frac{N}{2}\left(2^{\frac{1}{N}}-1\right) \frac{V(R)}{h(V(R) / 2)} \geq \frac{N}{2}\left(2^{\frac{1}{N}}-1\right) F(V(R)) .
\end{gathered}
$$

On combining (2.16)-(2.18), we get

$$
\gamma_{N, c}^{-1} R \leq F(V(R)) \leq \gamma_{N, c} R, \quad R>0,
$$

for a suitable $\gamma_{N, c}>1$. Thus owing to (2.14) and (2.15) we have

$$
\gamma^{-1} F^{(-1)}(R) \leq V(R) \leq \gamma F^{(-1)}(R)
$$

for a suitable $\gamma>1$.

Our claims (2.9) and (2.10) finally follow from (2.20) and again from (2.14)$(2.15)$.

Lemma 2.4. Under assumptions (1.26), (1.22) we have for $R>0$

$$
\gamma^{-1} \int_{B_{R}} \rho(d(x)) \mathrm{d} \mu \leq \rho(R) V(R) \leq \int_{B_{R}} \rho(d(x)) \mathrm{d} \mu .
$$

Proof. In fact simply by monotonicity of $\rho$ we have that

$$
\int_{B_{R}} \rho(d(x)) \mathrm{d} \mu \geq \rho(R) V(R) .
$$

Then we calculate, exploiting (2.3),

$$
\begin{aligned}
\int_{B_{R}} \rho(d(x)) \mathrm{d} \mu & =\int_{0}^{R} \rho(\tau) \frac{\mathrm{d} V}{\mathrm{~d} \tau}(\tau) \mathrm{d} \tau \leq C \int_{0}^{R}\left(\rho(R) R^{\alpha_{2}}\right) \tau^{-\alpha_{2}} \frac{\mathrm{d} V}{\mathrm{~d} \tau}(\tau) \mathrm{d} \tau \\
& =C \rho(R) R^{\alpha_{2}} \int_{0}^{R} \tau^{-\alpha_{2}} \frac{\mathrm{d} V}{\mathrm{~d} \tau}(\tau) \mathrm{d} \tau .
\end{aligned}
$$


The integral in (2.23) is handled by means of the change of variable $s=V(\tau)$, yielding

$$
\begin{aligned}
\int_{0}^{R} \tau^{-\alpha_{2}} \frac{\mathrm{d} V}{\mathrm{~d} \tau}(\tau) \mathrm{d} \tau & =\int_{0}^{V(R)} \frac{\mathrm{d} s}{V^{(-1)}(s)^{\alpha_{2}}}=\int_{0}^{V(R)} \frac{V^{(-1)}(s)^{p-\alpha_{2}}}{V^{(-1)}(s)^{p}} \mathrm{~d} s \\
& \leq V^{(-1)}(V(R))^{p-\alpha_{2}} \int_{0}^{V(R)} \frac{\mathrm{d} s}{V^{(-1)}(s)^{p}} \\
& \leq c^{-1} R^{p-\alpha_{2}} \frac{V(R)}{V^{(-1)}(V(R))^{p}}=c^{-1} R^{-\alpha_{2}} V(R) .
\end{aligned}
$$

Note that we used also (1.22).

Collecting (2.23) and (2.24) we obtain the claim.

Lemma 2.5. Under assumptions (1.22), (1.23), (1.25), (1.26), we have for all $s>$ $r>0$

$$
W(r):=\rho\left(R_{\rho}(r)\right) R_{\rho}(r)^{p} r^{-\frac{p-\alpha_{2}}{N-\alpha_{1}}} \leq \gamma W(s),
$$

for a suitable $\gamma>1$. In addition for $\lambda \geq 1, r>0$

$$
W(\lambda r) \leq \gamma_{1} \lambda^{d} W(r)
$$

where actually $\gamma_{1}=\tilde{c}^{-\left(p-\alpha_{1}\right) /\left(p-\alpha_{2}\right)}$ for $\tilde{c}$ as in $(2.5)$, and

$$
d=\frac{p-\alpha_{1}}{p-\alpha_{2}}-\frac{p-\alpha_{2}}{N-\alpha_{1}}>0 .
$$

Proof. We appeal to Lemma 2.1 with $f=W, r_{0}=V_{\rho}(1)>0$.

Let us begin by checking that $W$ is nondecreasing in $\left(V_{\rho}(1),+\infty\right)$; in this case $R_{\rho}(s)>1$. Write

$$
W(s)=\left[\rho\left(R_{\rho}(s)\right) R_{\rho}(s)^{\alpha_{2}}\right]\left[R_{\rho}(s) s^{-\frac{1}{N-\alpha_{1}}}\right]^{p-\alpha_{2}} .
$$

The first factor is nondecreasing by assumption (1.26). As to the second factor, set $R=R_{\rho}(s)$. Then the quantity in square brackets in such a factor equals

$$
R[\rho(R) V(R)]^{-\frac{1}{N-\alpha_{1}}}=\left[\rho(R) R^{\alpha_{1}}\right]^{-\frac{1}{N-\alpha_{1}}}\left[\frac{R^{N}}{V(R)}\right]^{\frac{1}{N-\alpha_{1}}} .
$$

Here, the first factor is nondecreasing by assumption (1.25); the second one is nondecreasing by assumption (1.23).

Next, we note that clearly two constants $C_{1}>C_{0}>0$ exist such that

$$
C_{0} s^{\frac{1}{N}} \leq R_{\rho}(s) \leq C_{1} s^{\frac{1}{N}}, \text { for } R_{\rho}(s) \leq 1 .
$$

Thus

$$
\rho(1) C_{0} s^{\frac{p}{N}-\frac{p-\alpha_{2}}{N-\alpha_{1}}} \leq W(s) \leq \rho(0) C_{1} s^{\frac{p}{N}-\frac{p-\alpha_{2}}{N-\alpha_{1}}} .
$$

It is easy to check that

$$
\beta=\frac{p}{N}-\frac{p-\alpha_{2}}{N-\alpha_{1}}=\frac{N \alpha_{2}-p \alpha_{1}}{N\left(N-\alpha_{1}\right)}>0,
$$

owing to our assumptions $N>p>\alpha_{2}>\alpha_{1}$. Thus for such a $\beta$ and $c_{0}, c_{1}$ given in (2.28) we have proved (2.1). Our claim (2.25) follows.

Our second claim (2.26) is a direct consequence of (1.25) and of (2.6). 
Lemma 2.6. $[2,30]$ Assume that $1<p<N$, and that (1.9), (1.20), (1.21), (1.22) hold true. Then for all $u \in W^{1, p}(M)$ we have

$$
\left(\int_{M}|u|^{p^{*}} \omega(V(d(x)))^{-p^{*}} \mathrm{~d} \mu\right)^{\frac{N-p}{N}} \leq C \int_{M}|\nabla u|^{p} \mathrm{~d} \mu,
$$

where $p^{*}=p N /(N-p)$ and $C>0$ is a suitable constant.

Proof. Our assumptions match the ones made in [2], when we show that the inequality

$$
\int_{0}^{k} s^{-p} h(s)^{p} \mathrm{~d} s \leq \gamma k^{-p+1} h(k), \quad k>0
$$

follows from our assumptions. Indeed, setting $R=V^{(-1)}(s)$, we have, with the notation of the proof of Lemma 2.3,

$$
\operatorname{sh}(s)^{-1}=V(R) h(V(R))^{-1}=F(V(R)),
$$

and therefore by $(2.20)$

$$
\gamma_{N, c}^{-1} V^{(-1)}(s) \leq \operatorname{sh}(s)^{-1} \leq \gamma_{N, c} V^{(-1)}(s), \quad s>0 .
$$

Then (2.29) follows from (2.31) and (1.22).

The weighted Sobolev inequality in [30] was proved under the assumption that the Ricci curvature is nonnegative and the volume growth is such to guarantee the hyperbolicity of the manifold. Instead in [2] we applied, to the same end, a symmetrization technique relying on an isoperimetrical inequality, as well as an assumption of $p$-hyperbolicity of $M$.

Next we prove the following Hardy inequality, needed below; see also the survey [12] on this subject.

Theorem 2.7. (Hardy inequality) Assume (1.9), (1.22), (1.24). For any $u \in W^{1, p}(M)$ we have

$$
\int_{M} \frac{|u|^{p}}{d(x)^{p}} \mathrm{~d} \mu \leq \gamma(N, p) \int_{M}|\nabla u|^{p} \mathrm{~d} \mu .
$$

Proof. We may assume $u \geq 0$. Introduce for $\lambda \geq 0$ the standard rearrangement function

$$
u^{*}(s)=\inf \left\{\lambda \mid \mu_{\lambda}<s\right\}, \quad \mu_{\lambda}=|\{x \in M|| u(x) \mid>\lambda\}| .
$$

We have

$$
\int_{M} \frac{u^{p}}{d(x)^{p}} \mathrm{~d} \mu \leq \int_{0}^{+\infty} u^{*}(s)^{p}\left[d(\cdot)^{-p}\right]^{*}(s) \mathrm{d} s .
$$

On the other hand

$$
\left|\left\{d(x)^{-p}>\lambda\right\}\right|=\left|B_{\lambda^{-\frac{1}{p}}}\right|=V\left(\lambda^{-\frac{1}{p}}\right) .
$$

Therefore (2.34) gives on integrating by parts

$$
\int_{M} \frac{u^{p}}{d(x)^{p}} \mathrm{~d} \mu \leq \int_{0}^{+\infty} \frac{u^{*}(s)^{p}}{V^{(-1)}(s)^{p}} \mathrm{~d} s=p \int_{0}^{+\infty} u^{*}(s)^{p-1}\left[-u_{s}^{*}(s)\right] \int_{0}^{s} \frac{\mathrm{d} \tau}{V^{(-1)}(\tau)^{p}} \mathrm{~d} s .
$$


Next we apply our assumption (1.22) in (2.35) and after applying Hölder inequality we arrive at

$$
\int_{0}^{+\infty} \frac{u^{*}(s)^{p}}{V^{(-1)}(s)^{p}} \mathrm{~d} s \leq \gamma\left(\int_{0}^{+\infty} \frac{u^{*}(s)^{p}}{V^{(-1)}(s)^{p}} \mathrm{~d} s\right)^{\frac{p-1}{p}}\left(\int_{0}^{+\infty}\left[-u_{s}^{*}(s)\right]^{p} \frac{s^{p}}{V^{(-1)}(s)^{p}} \mathrm{~d} s\right)^{\frac{1}{p}} .
$$

This immediately yields when we invoke (1.24)

$$
\begin{gathered}
\int_{0}^{+\infty} \frac{u^{*}(s)^{p}}{V^{(-1)}(s)^{p}} \mathrm{~d} s \leq \gamma \int_{0}^{+\infty}\left[-u_{s}^{*}(s)\right]^{p} \frac{s^{p}}{V^{(-1)}(s)^{p}} \mathrm{~d} s \\
\quad \leq \gamma \int_{0}^{+\infty}\left[-u_{s}^{*}(s)\right]^{p} h(s)^{p} \mathrm{~d} s \leq \gamma \int_{M}|\nabla u|^{p} \mathrm{~d} \mu,
\end{gathered}
$$

that is (2.32), by Polya-Szego principle (see [2]).

We state first an estimate where the density function $\rho$ does not appear.

Lemma 2.8. Let $u \in W^{1, p}(M), 0<r<q \leq N p /(N-p)$. Then

$$
\int_{M}|u|^{q} \mathrm{~d} \mu \leq \gamma \omega\left(S_{q}\right)^{q} S_{q}^{1+\frac{q}{N}-\frac{q}{p}}\|\nabla u\|_{L^{p}(M)}^{q},
$$

where

$$
S_{q}=\left(\int_{M}|u|^{r} \mathrm{~d} \mu\right)^{\frac{q}{q-r}}\left(\int_{M}|u|^{q} \mathrm{~d} \mu\right)^{-\frac{r}{q-r}} .
$$

Proof. We confine ourselves to the case $q \leq p$, which is the one of our interest here. The case $q>p$ can be proved reasoning as in [5].

Introduce the standard rearrangement function as in (2.33). Then write for convenience of notation

$$
P_{s}=\int_{M}|u(x)|^{s} \mathrm{~d} \mu, \quad s>0 .
$$

We have for a $k>0$ to be selected presently

$$
\begin{aligned}
P_{q} & =\int_{0}^{\mu_{0}} u^{*}(s)^{q} \mathrm{~d} s \leq \gamma(q) \int_{0}^{\mu_{k}}\left(u^{*}(s)-k\right)^{q} \mathrm{~d} s+\gamma(q) k^{q} \mu_{k}+\int_{\mu_{k}}^{\mu_{0}} u^{*}(s)^{q} \mathrm{~d} s \\
& =: I_{1}+I_{2}+I_{3} .
\end{aligned}
$$

Next we invoke Chebyshev inequality

$$
k^{r} \mu_{k} \leq P_{r}
$$

to bound

$$
I_{2}+I_{3} \leq \gamma \mu_{k}^{1-\frac{q}{r}} P_{r}^{\frac{q}{r}}+k^{q-r} \int_{\mu_{k}}^{\mu_{0}} u^{*}(s)^{r} \mathrm{~d} s \leq \gamma \mu_{k}^{1-\frac{q}{r}} P_{r}^{\frac{q}{r}}=\frac{1}{2} P_{q} .
$$

The last equality in (2.41) is our choice of $k$, which amounts to $\mu_{k}=\gamma S_{q}$. Note that we may assume $\mu_{0}$ as large as necessary, by approximating $u$ while keeping all the involved integral quantities stable. Thus we can safely assume that such a value of 
$k$ exists. Hence we absorb $I_{2}+I_{3}$ into the left hand side of (2.40). We then reason as in [40] to obtain

$$
\begin{aligned}
P_{q} & \leq \gamma \int_{0}^{\mu_{k}}\left(u^{*}(s)-k\right)^{q} \mathrm{~d} s \leq \gamma \mu_{k}^{1-\frac{q}{p}}\left(\int_{0}^{\mu_{k}}\left(u^{*}(s)-k\right)^{p} \mathrm{~d} s\right)^{\frac{q}{p}} \\
& \leq \gamma \mu_{k}^{1-\frac{q}{p}}\left(\int_{0}^{\mu_{k}}\left[-u_{s}^{*}(s)\right]^{p} h(s)^{p}\left[s h(s)^{-1}\right]^{p} \mathrm{~d} s\right)^{\frac{q}{p}} \\
& \leq \gamma \mu_{k}^{1-\frac{q}{p}}\left[\mu_{k} h\left(\mu_{k}\right)^{-1}\right]^{q}\left(\int_{M}|\nabla u|^{p} \mathrm{~d} \mu\right)^{\frac{q}{p}} .
\end{aligned}
$$

We have exploited here the fact that $t \mapsto t h(t)^{-1}$ is increasing as a consequence of our assumption that $\omega$ is nondecreasing.

Finally (2.38) follows from (2.42) and from our choice $\mu_{k}=\gamma S_{q}$.

Corollary 2.9. Let $u \in W^{1, p}(M)$ and $0<r<p$. Then

$$
\begin{aligned}
& \int_{M}|u|^{p} \mathrm{~d} \mu \leq \gamma \omega(\mu(\operatorname{supp} u))^{\frac{p N(p-r)}{N(p-r)+r p}}\left(\int_{M}|u|^{r} \mathrm{~d} \mu\right)^{\frac{p^{2}}{N(p-r)+r p}} \\
& \quad \times\left(\int_{M}|\nabla u|^{p} \mathrm{~d} \mu\right)^{\frac{N(p-r)}{N(p-r)+r p}} .
\end{aligned}
$$

Proof. We select $q=p$ in Lemma 2.8. The statement follows from an elementary computation, when we also bound by means of Hölder's inequality

$$
S_{q} \leq\left[\mu(\operatorname{supp} u)^{1-\frac{r}{p}}\left(\int_{M}|u|^{p} \mathrm{~d} \mu\right)^{\frac{r}{p}}\right]^{\frac{p}{p-r}}\left(\int_{M}|u|^{p} \mathrm{~d} \mu\right)^{-\frac{r}{p-r}}=\mu(\operatorname{supp} u)
$$

Next we state some weighted estimates where the estimated norms involve the weight $\rho$. We denote in the following for $k>0$

$$
\mu_{\rho}(A)=\int_{A} \rho(d(x)) \mathrm{d} \mu, \quad \nu_{\rho}(k)=\mu_{\rho}(\{u>k\}) .
$$

Lemma 2.10. (Faber-Krahn type estimate) Assume that $1<p<N$, and that the assumptions of Lemma 2.6 and of Theorem 2.7 hold true. We further assume (1.26), (1.28).

Then for all $u \in W^{1, p}(M), k>0$ we have

$$
\int_{\{u>k\}} \rho(d(x))(u-k)^{p} \mathrm{~d} \mu \leq \gamma \rho\left(R_{\rho}\left(\nu_{\rho}(k)\right)\right) R_{\rho}\left(\nu_{\rho}(k)\right)^{p} \int_{\{u>k\}}|\nabla u|^{p} \mathrm{~d} \mu .
$$


Proof. We split the integral between $B(R)$ and its complement; let $A_{k}=\{u>k\}$. We first have from Lemma 2.6,

$$
\begin{aligned}
\int_{A_{k} \backslash B_{R}} \rho(d(x))(u-k)^{p} \mathrm{~d} \mu \leq & \left(\int_{A_{k}}(u-k)^{p^{*}} \omega(V(d(x)))^{-p^{*}} \mathrm{~d} \mu\right)^{\frac{N-p}{N}} \\
& \times\left(\int_{A_{k} \backslash B_{R}} \rho(d(x))^{\frac{N}{p}} \omega(V(d(x)))^{N} \mathrm{~d} \mu\right)^{\frac{p}{N}} \\
\leq & \gamma \rho(R)^{\frac{N-p}{N}} \omega(V(R))^{p} \nu_{\rho}(k)^{\frac{p}{N}}\left(\int_{A_{k}}|\nabla u|^{p} \mathrm{~d} \mu\right) \\
\leq & \gamma \rho(R)^{\frac{N-p}{N}} R^{p} V(R)^{-\frac{p}{N}} \nu_{\rho}(k)^{\frac{p}{N}}\left(\int_{A_{k}}|\nabla u|^{p} \mathrm{~d} \mu\right)=: I_{1} .
\end{aligned}
$$

We used assumption (1.28) and the estimate (2.19), at least for $R>s_{0}$ as in (1.28). For $R \leq s_{0}$ we simply note that

$$
c \leq \rho(s) \omega(V(s))^{p^{*}} \leq c^{-1}, \quad 0<s \leq s_{0},
$$

for a suitable $0<c<1$, as $\omega(0+)>0$ due to the locally Euclidean behavior of the Riemannian metric.

Next we have by means of Hardy inequality (2.32), and also by taking into account (2.3), that

$$
\begin{aligned}
\int_{A_{k} \cap B(R)} \rho(d(x))(u-k)^{p} \mathrm{~d} \mu & =\int_{A_{k} \cap B(R)} \rho(d(x)) d(x)^{p} d(x)^{-p}(u-k)^{p} \mathrm{~d} \mu \\
& \leq \gamma \rho(R) R^{p} \int_{A_{k}}|\nabla u|^{p} \mathrm{~d} \mu=: I_{2} .
\end{aligned}
$$

Finally we select $R$ so that, essentially, $I_{1}$ and $I_{2}$ contribute the same quantity, i.e.,

$$
\rho(R)^{\frac{N-p}{N}} R^{p} V(R)^{-\frac{p}{N}} \nu_{\rho}(k)^{\frac{p}{N}}=\rho(R) R^{p},
$$

or as one can immediately see

$$
V_{\rho}(R)=\nu_{\rho}(k)
$$

The claim follows.

Lemma 2.11. Assume that $1<p<N$, and that the assumptions of Lemma 2.6, Theorem 2.7 and (1.28) hold true. Then for all $u \in W^{1, p}(M)$ and $k>0$ we have

$$
\begin{aligned}
& \int_{\{u>k\}} \rho(d(x))(u-k)^{s} \mathrm{~d} \mu \\
& \quad \leq \gamma\left[\rho\left(R_{\rho}\left(\nu_{\rho}(k)\right)\right) R_{\rho}\left(\nu_{\rho}(k)\right)^{p}\right]^{\frac{s}{p}} \nu_{\rho}(k)^{1-\frac{s}{p}}\left(\int_{\{u>k\}}|\nabla u|^{p} \mathrm{~d} \mu\right)^{\frac{s}{p}},
\end{aligned}
$$

provided $p<s<p^{*}$ and that, in addition, for a given $C>0$

$$
\rho(R) R^{N-\frac{s(N-p)}{p}} \leq C \rho\left(R_{1}\right) R_{1}^{N-\frac{s(N-p)}{p}}, \quad R_{1}>R>0 .
$$


Proof. As in the proof of Lemma 2.10, we split the integral between $B(R)$ and its complement; let $A_{k}=\{u>k\}$ and $\nu_{\rho}(k)$ be defined as in (2.45).

We first have from Hölder inequality, Lemma 2.6, and from assumption (1.28),

$$
\begin{aligned}
\int_{A_{k} \backslash B_{R}} \rho(d(x))(u-k)^{s} \mathrm{~d} \mu \leq & \left(\int_{A_{k} \backslash B_{R}} \omega(V(d(x)))^{-p^{*}}(u-k)^{p^{*}} \mathrm{~d} \mu\right)^{\frac{s}{p^{*}}} \\
& \times\left(\int_{A_{k} \backslash B_{R}} \rho(d(x))^{\frac{p^{*}}{p^{*}-s}} \omega(V(d(x)))^{\frac{p^{*} s}{p^{*}-s}} \mathrm{~d} \mu\right)^{1-\frac{s}{p^{*}}} \\
\leq & \gamma \rho(R)^{\frac{s}{p^{*}}} \omega(V(R))^{s} \nu_{\rho}(k)^{1-\frac{s}{p^{*}}}\left(\int_{A_{k}}|\nabla u|^{p} \mathrm{~d} \mu\right)^{\frac{s}{p}} \\
\leq & \gamma \rho(R)^{\frac{s}{p^{*}}} R^{s} V(R)^{-\frac{s}{N}} \nu_{\rho}(k)^{1-\frac{s}{p^{*}}}\left(\int_{A_{k}}|\nabla u|^{p} \mathrm{~d} \mu\right)^{\frac{s}{p}}=: I_{1} .
\end{aligned}
$$

We have used in last inequality our assumption (1.24).

Next we apply again Hölder inequality to get

$$
\begin{aligned}
& \int_{A_{k} \cap B_{R}} \rho(d(x))(u-k)^{s} \mathrm{~d} \mu \leq\left(\int_{A_{k} \cap B_{R}} d(x)^{-p}(u-k)^{p} \mathrm{~d} \mu\right)^{\frac{p^{*}-s}{p^{*}-p}} \\
& \times\left(\int _ { A _ { k } \cap B _ { R } } \left[\rho(d(x)) d(x)^{\left.\left.N-(N-p) \frac{s}{p}\right]^{\frac{p^{*}-p}{s-p}}(u-k)^{p^{*}} \mathrm{~d} \mu\right)^{\frac{s-p}{p^{*}-p}} .}\right.\right.
\end{aligned}
$$

The second factor in (2.54) is majorized, owing to our assumption (2.52), by

$$
\begin{aligned}
& \gamma \omega(V(R))^{\frac{N(s-p)}{p}} \rho(R) R^{N-(N-p) \frac{s}{p}}\left(\int_{A_{k} \cap B_{R}}(u-k)^{p^{*}} \omega(V(d(x)))^{-p^{*}} \mathrm{~d} \mu\right)^{\frac{s-p}{p^{*}-p}} \\
& \leq \gamma \omega(V(R))^{\frac{N(s-p)}{p}} \rho(R) R^{N-(N-p) \frac{s}{p}}\left(\int_{A_{k}}|\nabla u|^{p} \mathrm{~d} \mu\right)^{\frac{N(s-p)}{p^{2}}}
\end{aligned}
$$

when we apply also Lemma 2.6. The first integral in (2.54) is bounded with the help of Hardy inequality (2.32), to obtain finally

$$
\begin{aligned}
& \int_{A_{k} \cap B_{R}} \rho(d(x))(u-k)^{s} \mathrm{~d} \mu \\
& \leq \gamma \omega(V(R))^{\frac{N(s-p)}{p}} \rho(R) R^{N-(N-p) \frac{s}{p}}\left(\int_{A_{k}}|\nabla u|^{p} \mathrm{~d} \mu\right)^{\frac{s}{p}} \\
& \leq \gamma R^{\frac{N(s-p)}{p}} V(R)^{-\frac{s-p}{p}} \rho(R) R^{N-(N-p) \frac{s}{p}}\left(\int_{A_{k}}|\nabla u|^{p} \mathrm{~d} \mu\right)^{\frac{s}{p}}=: I_{2} .
\end{aligned}
$$

In last inequality we used again (1.24).

We require that $I_{1}$ and $I_{2}$ contribute essentially the same quantity, that is we define $R$ by means of

$$
\rho(R)^{\frac{s}{p^{*}}} R^{s} V(R)^{-\frac{s}{N}} \nu_{\rho}(k)^{1-\frac{s}{p^{*}}}=R^{\frac{N(s-p)}{p}} V(R)^{-\frac{s-p}{p}} \rho(R) R^{N-(N-p) \frac{s}{p}} .
$$

It is easily seen that this is equivalent to $V_{\rho}(R)=\nu_{\rho}(k)$, i.e., $R=R_{\rho}\left(\nu_{\rho}(k)\right)$. With this choice of $R$ it is trivial to check that, e.g., the right hand side of (2.57) equals $\rho(R)^{s / p} R^{s} \nu_{\rho}(k)^{1-s / p}$, proving the claim. 
Remark 2.12. Concerning assumption (2.52) we note that the exponent of $R$ in it, that is $N-s(N-p) / p$, tends to $p-$ as $s \rightarrow p+$. Hence for suitable $s$ in such a range, (2.52) is a consequence of (1.26) and of Lemma 2.1.

Let us also note for later use the following consequence of (2.52): rewrite the right hand side of $(2.57)$, that is $\rho(R) R^{s} V(R)^{(p-s) / p}$ as

$$
\rho(R) R^{N-\frac{s(N-p)}{p}}\left(\frac{R^{N}}{V(R)}\right)^{\frac{s-p}{p}} .
$$

The last factor above is nondecreasing owing to our assumption (1.23); thus, owing to $(2.52)$ we have

$$
\rho(R) R^{s} V(R)^{\frac{p-s}{p}} \leq C \rho\left(R_{1}\right) R_{1}^{s} V\left(R_{1}\right)^{\frac{p-s}{p}}, \quad 0<R<R_{1},
$$

for $C>0$ as in (2.52).

In the following we let for the sake of notational simplicity for $p>r>0$

$$
E_{r}=\int_{M} \rho(d(x))|u|^{r} \mathrm{~d} \mu, \quad S=\frac{E_{r}^{\frac{p}{p-r}}}{E_{p}^{\frac{r}{p-r}}} .
$$

Lemma 2.13. (Sobolev-Gagliardo-Nirenberg) Assume that $1<p<N$, and that the assumptions of Lemma 2.10 hold true. Assume further that $0<r<p$. Then we have for all $u \in W^{1, p}(M)$

$$
E_{p} \leq \gamma \rho\left(R_{\rho}(S)\right) R_{\rho}(S)^{p} \int_{M}|\nabla u|^{p} \mathrm{~d} \mu .
$$

Proof. We begin by splitting, for a $k>0$ to be chosen,

$$
E_{p}=\int_{\{|u|>k\}} \rho(d(x))|u|^{p} \mathrm{~d} \mu+\int_{\{|u| \leq k\}} \rho(d(x))|u|^{p} \mathrm{~d} \mu=: J_{1}+J_{2} .
$$

We first bound, on using (2.46) and Chebyshev inequality,

$$
\begin{aligned}
J_{1} & \leq 2^{p-1} \int_{\{|u|>k\}} \rho(d(x))(|u|-k)^{p} \mathrm{~d} \mu+2^{p-1} k^{p} \nu_{\rho}(k) \\
& \leq \gamma \rho\left(R_{\rho}\left(\nu_{\rho}(k)\right)\right) R_{\rho}\left(\nu_{\rho}(k)\right)^{p} \int_{M}|\nabla u|^{p} \mathrm{~d} \mu+2^{p-1} k^{p-r} E_{r} .
\end{aligned}
$$

Then we get obviously

$$
J_{2} \leq k^{p-r} E_{r} .
$$

Collecting (2.61)-(2.63) we obtain, after a further use of Chebyshev inequality,

$$
E_{p} \leq \gamma \rho\left(R_{\rho}\left(k^{-r} E_{r}\right)\right) R_{\rho}\left(k^{-r} E_{r}\right)^{p} \int_{M}|\nabla u|^{p} \mathrm{~d} \mu+\left(2^{p-1}+1\right) k^{p-r} E_{r} .
$$

We select next $k$ so that

$$
\rho\left(R_{\rho}\left(k^{-r} E_{r}\right)\right) R_{\rho}\left(k^{-r} E_{r}\right)^{p} \int_{M}|\nabla u|^{p} \mathrm{~d} \mu=k^{p-r} E_{r} .
$$

Then we have first

$$
E_{p} \leq \gamma k^{p-r} E_{r}
$$


yielding at once

$$
E_{r} k^{-r} \leq \gamma S .
$$

On appealing again to (2.64) and (2.65), together with (2.67) we obtain, recalling $(2.6)$

$$
\begin{aligned}
E_{p} & \leq \gamma \rho\left(R_{\rho}(\gamma S)\right) R_{\rho}(\gamma S)^{p} \int_{M}|\nabla u|^{p} \mathrm{~d} \mu \\
& \leq \gamma \rho\left(R_{\rho}(S)\right) R_{\rho}(S)^{p} \int_{M}|\nabla u|^{p} \mathrm{~d} \mu .
\end{aligned}
$$

Lemma 2.14. Assume that $1<p<s<N$, and that the assumptions of Lemma 2.11 hold true. Assume further that $0<r<p$. Then we have for all $u \in W^{1, p}(M)$

$$
E_{s} \leq \gamma\left[\rho\left(R_{\rho}(\Sigma)\right) R_{\rho}(\Sigma)^{p}\right]^{\frac{s}{p}} \Sigma^{1-\frac{s}{p}}\left(\int_{M}|\nabla u|^{p} \mathrm{~d} \mu\right)^{\frac{s}{p}}
$$

where $\Sigma=E_{r}^{s /(s-r)} E_{s}^{-r /(s-r)}$.

Proof. As in the proof of Lemma 2.13, we begin by splitting for a $k>0$ to be chosen,

$$
E_{s}=\int_{\{|u|>k\}} \rho(d(x))|u|^{s} \mathrm{~d} \mu+\int_{\{|u| \leq k\}} \rho(d(x))|u|^{s} \mathrm{~d} \mu=: J_{1}+J_{2} .
$$

Then we bound $J_{1}$ essentially as we did in (2.62), but applying (2.51) rather than (2.46), and $J_{2}$ following the direct approach of (2.63). We get, collecting such estimates, after a further use of Chebyshev inequality,

$$
\begin{aligned}
E_{s} \leq & \gamma\left[\rho\left(R_{\rho}\left(k^{-r} E_{r}\right)\right) R_{\rho}\left(k^{-r} E_{r}\right)^{p}\right]^{\frac{s}{p}}\left(k^{-r} E_{r}\right)^{1-\frac{s}{p}}\left(\int_{M}|\nabla u|^{p} \mathrm{~d} \mu\right)^{\frac{s}{p}} \\
& +\left(2^{s-1}+1\right) k^{s-r} E_{r} .
\end{aligned}
$$

We have made use of (2.58) here.

Next we choose $k$ so that

$$
\left[\rho\left(R_{\rho}\left(k^{-r} E_{r}\right)\right) R_{\rho}\left(k^{-r} E_{r}\right)^{p}\right]^{\frac{s}{p}}\left(k^{-r} E_{r}\right)^{1-\frac{s}{p}}\left(\int_{M}|\nabla u|^{p} \mathrm{~d} \mu\right)^{\frac{s}{p}}=k^{s-r} E_{r},
$$

implying first that

$$
E_{s} \leq \gamma k^{s-r} E_{r}
$$

so that

$$
E_{r} k^{-r} \leq \gamma \Sigma
$$

The proof then is concluded by substituting (2.74) in (2.71), on recalling (2.72), and (2.58), too.

Corollary 2.15. Under the assumptions and with the notation of Lemma 2.13 we have for the function $W$ defined in (2.25)

$$
E_{p} \leq \gamma E_{r}^{\frac{p\left(p-\alpha_{2}\right)}{\mathcal{H}(r)}} W(S)^{\frac{(p-r)\left(N-\alpha_{1}\right)}{\mathcal{H}(r)}}\left(\int_{M}|\nabla u|^{p} \mathrm{~d} \mu\right)^{\frac{(p-r)\left(N-\alpha_{1}\right)}{\mathcal{H}(r)}},
$$


where $\mathcal{H}(r)=(p-r)\left(N-\alpha_{1}\right)+r\left(p-\alpha_{2}\right)$ for $r>0$.

Proof. We only need to multiply and divide the right hand side of (2.60) by $S^{\left(p-\alpha_{2}\right) /\left(N-\alpha_{1}\right)}$; then $(2.75)$ follows after some elementary algebra, when we recall the definitions of $S$ and of $W$.

Again by simple algebra we infer also the following result.

Corollary 2.16. Under the assumptions and with the notation of Lemma 2.14 we have for the function $W$ defined in (2.25)

$$
E_{s} \leq \gamma E_{r}^{\frac{\mathcal{H}(s)}{\mathcal{H}(r)}} W(\Sigma)^{\frac{(s-r)\left(N-\alpha_{1}\right)}{\mathcal{H}(r)}}\left(\int_{M}|\nabla u|^{p} \mathrm{~d} \mu\right)^{\frac{(s-r)\left(N-\alpha_{1}\right)}{\mathcal{H}(r)}}
$$

where $\mathcal{H}$ is as in Corollary 2.15 .

We'll use the following inequalities. The set $C_{0}^{1}(M)$ denotes the $C^{1}(M)$ functions with compact support.

Lemma 2.17. Let $u$ be a solution of (1.1) and (1.2), and let $\theta>0$, with $\theta>2-m$ if $m<1, k>h>0,0<\tau_{2}<\tau_{1}$ be given. Then

$$
\begin{aligned}
& \sup _{\tau_{1}<\tau<t} \int_{M}(u-k)_{+}^{1+\theta} \rho \mathrm{d} \mu+\int_{\tau_{1}}^{t} \int_{M}\left|\nabla(u-k)_{+}^{\frac{p+m+\theta-2}{p}}\right| p d \mu \mathrm{d} \tau \\
& \leq \gamma \frac{H(h, k)}{\tau_{1}-\tau_{2}} \int_{\tau_{2}}^{t} \int_{M}(u-h)_{+}^{1+\theta} \rho \mathrm{d} \mu \mathrm{d} \tau,
\end{aligned}
$$

provided the right hand side in (2.77) is finite. Here $H(h, k)=(k /(k-h))^{(m-1)_{-}}$.

Lemma 2.18. Let $u$ be a solution of (1.1) and (1.2), and let $\theta \geq p-1$. Let $\zeta \in$ $C_{0}^{1}(M), 0 \leq \zeta \leq 1$. Then

$$
\begin{aligned}
& \sup _{0<\tau<t} \int_{M}(u \zeta)^{1+\theta} \rho \mathrm{d} \mu+\int_{0}^{t} \int_{M}\left|\nabla(u \zeta)^{\frac{p+m+\theta-2}{p}}\right|^{p} \mathrm{~d} \mu \mathrm{d} \tau \\
& \leq \gamma\left\{\int_{0}^{t} \int_{M}|\nabla \zeta|^{p} u^{p+m+\theta-2} \mathrm{~d} \mu \mathrm{d} \tau+\int_{M}\left(u_{0} \zeta\right)^{1+\theta} \mathrm{d} \mu\right\},
\end{aligned}
$$

provided the right hand side in (2.78) is finite.

The proofs of Lemmas 2.17 and 2.18 are standard and we omit them.

\section{Proof of the Sup Estimate in the Case $p+m-3>0$}

For $k>0$ to be selected later, and for $\theta>0$ as in Lemma 2.17, we define for $n \geq 0$

$$
\begin{aligned}
& k_{n}=k\left(1-2 \sigma+2^{-n} \sigma\right), \quad r=\frac{p}{p+m+\theta-2}, \\
& s=r(1+\theta)<p, \quad v_{n}=\left(u-k_{n}\right)_{+}^{\frac{1}{r}}, \quad \tau_{n}=\frac{t}{2}\left(1-2 \sigma+2^{-n} \sigma\right) .
\end{aligned}
$$

Here $\sigma \in(1,1 / 4)$ is fixed. From Lemma 2.17 we infer

$$
\sup _{\tau_{n}<\tau<t} \int_{M} \rho v_{n}^{s} \mathrm{~d} \mu+\int_{\tau_{n}}^{t} \int_{M}\left|\nabla v_{n}\right|^{p} \mathrm{~d} \mu \mathrm{d} \tau \leq \gamma \frac{2^{n \ell}}{\sigma^{\ell} t} \int_{\tau_{n+1}}^{t} \int_{M} \rho v_{n+1}^{s} \mathrm{~d} \mu \mathrm{d} \tau,
$$


for $\ell=1+(m-1)_{-}$. From Hölder inequality it follows

$$
\int_{M} \rho v_{n+1}^{s} \mathrm{~d} \mu \leq\left(\int_{M} \rho v_{n+1}^{p} \mathrm{~d} \mu\right)^{\frac{s-r}{p-r}}\left(\int_{M} \rho v_{n+1}^{r} \mathrm{~d} \mu\right)^{\frac{p-s}{p-r}},
$$

whence, on applying Corollary 2.15 to bound the first integral on the right hand side of (3.2), we get

$$
\begin{aligned}
& \int_{M} \rho v_{n+1}^{s} \mathrm{~d} \mu \leq \gamma W(S)^{\frac{(s-r)\left(N-\alpha_{1}\right)}{\mathcal{H}(r)}}\left(\int_{M} \rho v_{n+1}^{r} \mathrm{~d} \mu\right)^{\frac{p-s}{p-r}+\frac{(s-r) p\left(p-\alpha_{2}\right)}{(p-r) \mathcal{H}(r)}} \\
& \quad \times\left(\int_{M}\left|\nabla v_{n+1}\right|^{p} \mathrm{~d} \mu\right)^{\frac{(s-r)\left(N-\alpha_{1}\right)}{\mathcal{H}(r)}} .
\end{aligned}
$$

here $S$ is defined as in (2.59). Note that by appealing again to Hölder inequality we obtain, with the notation introduced in (2.45)

$$
S \leq \int_{\left\{v_{n+1}>0\right\}} \rho \mathrm{d} \mu \leq \nu_{\rho}\left(k_{\infty}\right)=\mu_{\rho}\left(\left\{u(\tau)>k_{\infty}\right\}\right), \quad k_{\infty}=k(1-2 \sigma) .
$$

Next we integrate in time the estimate (3.3), and after a further application of Hölder inequality we bound the right hand side of (3.1) by

$$
\begin{aligned}
\gamma \frac{2^{n \ell}}{\sigma^{\ell} t} & \int_{\tau_{n+1}}^{t}\left(\int_{M} \rho v_{n+1}^{r} \mathrm{~d} \mu\right)^{\frac{p-s}{p-r}+\frac{(s-r) p\left(p-\alpha_{2}\right)}{(p-r) \mathcal{H}(r)}}\left(\int_{M}\left|\nabla v_{n+1}\right|^{p} \mathrm{~d} \mu\right)^{\frac{(s-r)\left(N-\alpha_{1}\right)}{\mathcal{H}(r)}} \mathrm{d} \tau \\
& \times \sup _{\tau_{n+1}<\tau<t} W\left(\left\{u(\tau)>k_{\infty}\right\}\right)^{\frac{(s-r)\left(N-\alpha_{1}\right)}{\mathcal{H}(r)}} \\
\leq & \frac{2^{n \ell}}{\sigma^{\ell}} t^{-\frac{(s-r)\left(N-\alpha_{1}\right)}{\mathcal{H}(r)}}\left(\int_{\tau_{n+1}}^{t} \int_{M}\left|\nabla v_{n+1}\right|^{p} \mathrm{~d} \mu \mathrm{d} \tau\right)^{\frac{(s-r)\left(N-\alpha_{1}\right)}{\mathcal{H}(r)}} \\
& \times \sup _{\tau_{n+1}<\tau<t}\left(\int_{M} \rho v_{n+1}^{r} \mathrm{~d} \mu\right)^{\frac{\mathcal{H}(s)}{\mathcal{H}(r)}} \sup _{\tau_{n+1}<\tau<t} W\left(\left\{u(\tau)>k_{\infty}\right\}\right)^{\frac{(s-r)\left(N-\alpha_{1}\right)}{\mathcal{H}(r)}}
\end{aligned}
$$

Let us define for the sake of notational simplicity $\xi=\mathcal{H}(r) /\left[(s-r)\left(N-\alpha_{1}\right)\right]$. Next we invoke Young inequality to bound the right hand side of (3.5) by

$$
\begin{aligned}
& \varepsilon \int_{\tau_{n+1}}^{t} \int_{M}\left|\nabla v_{n+1}\right|^{p} \mathrm{~d} \mu \mathrm{d} \tau+\gamma \frac{b^{n}}{\sigma^{\frac{\ell \xi}{\xi-1}}} \varepsilon^{-\frac{1}{\xi-1}} t^{-\frac{(s-r)\left(N-\alpha_{1}\right)}{(p-s)\left(N-\alpha_{1}\right)+r\left(p-\alpha_{2}\right)}} \\
& \quad \times \sup _{\tau_{n+1}<\tau<t} W\left(\left\{u(\tau)>k_{\infty}\right\}\right)^{\frac{(s-r)\left(N-\alpha_{1}\right)}{(p-s)\left(N-\alpha_{1}\right)+r\left(p-\alpha_{2}\right)}} \\
& \quad \times \sup _{\tau_{n+1}<\tau<t}\left(\int_{M} \rho v_{n+1}^{r} \mathrm{~d} \mu\right)^{\frac{\mathcal{H}(s)}{(p-s)\left(N-\alpha_{1}\right)+r\left(p-\alpha_{2}\right)}}
\end{aligned}
$$

where $b=2^{\ell \xi /(\xi-1)}$. On combining (3.1)-(3.6), and denoting

$$
I_{n}=\sup _{\tau_{n}<\tau<t} \int_{M} \rho v_{n}(\tau)^{s} \mathrm{~d} \mu+\int_{\tau_{n}}^{t} \int_{M}\left|\nabla v_{n}\right|^{p} \mathrm{~d} \mu \mathrm{d} \tau
$$


we get the recursive inequality

$$
\begin{aligned}
I_{n} \leq & \varepsilon I_{n+1}+\gamma \frac{b^{n}}{\sigma \frac{\ell \xi}{\xi-1}} \varepsilon^{-\frac{1}{\xi-1}} t^{-\frac{(s-r)\left(N-\alpha_{1}\right)}{(p-s)\left(N-\alpha_{1}\right)+r\left(p-\alpha_{2}\right)}} \\
& \times \sup _{\tau_{\infty}<\tau<t} W\left(\left\{u(\tau)>k_{\infty}\right\}\right)^{\frac{(s-r)\left(N-\alpha_{1}\right)}{(p-s)\left(N-\alpha_{1}\right)+r\left(p-\alpha_{2}\right)}} \\
& \times \sup _{\tau_{\infty}<\tau<t}\left(\int_{M} \rho v_{\infty}^{r} \mathrm{~d} \mu\right)^{\frac{\mathcal{H}(s)}{(p-s)\left(N-\alpha_{1}\right)+r\left(p-\alpha_{2}\right)}}
\end{aligned}
$$

where $\tau_{\infty}=t(1-2 \sigma) / 2, v_{\infty}=\left(u-k_{\infty}\right)_{+}^{1 / r}$.

A standard iteration process then implies that, when we select e.g., $\varepsilon=1 /(2 b)$,

$$
\begin{aligned}
& \sup _{\tau_{0}<\tau<t} \int_{M} \rho\left(u(\tau)-k_{0}\right)_{+}^{1+\theta} \mathrm{d} \mu \leq \gamma \sigma^{-\frac{\ell \xi}{\xi-1}} t^{-\frac{\theta\left(N-\alpha_{1}\right)}{\eta}} \\
& \quad \times \sup _{\tau_{\infty}<\tau<t} W\left(\left\{u(\tau)>k_{\infty}\right\}\right)^{\frac{\theta\left(N-\alpha_{1}\right)}{\eta}} \\
& \quad \times \sup _{\tau_{\infty}<\tau<t}\left(\int_{M} \rho\left(u(\tau)-k_{\infty}\right)_{+} \mathrm{d} \mu\right)^{1+\frac{\theta\left(p-\alpha_{2}\right)}{\eta}},
\end{aligned}
$$

where we have computed

$$
\begin{aligned}
& \frac{(s-r)\left(N-\alpha_{1}\right)}{(p-s)\left(N-\alpha_{1}\right)+r\left(p-\alpha_{2}\right)}=\frac{\theta\left(N-\alpha_{1}\right)}{\eta}, \\
& \frac{\mathcal{H}(s)}{(p-s)\left(N-\alpha_{1}\right)+r\left(p-\alpha_{2}\right)}=1+\frac{\theta\left(p-\alpha_{2}\right)}{\eta}, \\
& \eta=\left(N-\alpha_{1}\right)(p+m-3)+p-\alpha_{2} .
\end{aligned}
$$

Next we set in $(3.9)$

$$
\begin{aligned}
\tau_{\infty} & =t_{j}=\frac{t}{2}\left(1-2^{-j-2}\right), \quad \tau_{0}=t_{j+1}, \quad \sigma=2^{-j-3}, \\
k_{\infty} & =h_{j}=k\left(1-2^{-j-2}\right), \quad k_{0}=h_{j+1}, \\
y_{j} & =\sup _{t_{j}<\tau<t} \int_{M} \rho\left(u(\tau)-h_{j}\right)_{+} \mathrm{d} \mu, \quad j \geq 0 .
\end{aligned}
$$

Note that by Chebyshev inequality we obtain there

$$
W\left(\mu_{\rho}\left(\left\{u(\tau)>h_{j}\right\}\right)\right) \leq W\left(2 h_{j}^{-1} \int_{M} \rho u(\tau) \mathrm{d} \mu\right) \leq \gamma W\left(k^{-1} U\right),
$$

when we employ also Lemma 2.5 and

$$
U:=\sup _{\frac{t}{2}<\tau<t} \int_{M} \rho u(\tau) \mathrm{d} \mu \geq y_{j}, \quad j \geq 0 .
$$

By the same token, the left hand side of (3.9) is bounded from below by

$$
k^{\theta} 2^{-(j+4) \theta} y_{j+2} \text {. }
$$

Thus we infer for $Y_{i}=y_{2 i}, i \geq 0$,

$$
Y_{i+1} \leq \gamma 4^{\left(\frac{\ell \xi}{\xi-1}+\theta\right) i} k^{-\theta} t^{-\frac{\theta\left(N-\alpha_{1}\right)}{\eta}} W\left(k^{-1} U\right)^{\frac{\theta\left(N-\alpha_{1}\right)}{\eta}} Y_{i}^{1+\frac{\theta\left(p-\alpha_{2}\right)}{\eta}} .
$$


According to the classical result [26, Lemma 5.6, Chapt. II], we get that $Y_{i} \rightarrow 0$ as $i \rightarrow+\infty$ provided

$$
k^{-1} t^{-\frac{N-\alpha_{1}}{\eta}} W\left(k^{-1} U\right)^{\frac{N-\alpha_{1}}{\eta}} U^{\frac{p-\alpha_{2}}{\eta}} \leq \gamma_{0},
$$

for a suitably small constant $\gamma_{0}>0$ depending on the parameters in (3.12).

When we recall the definition of the function $W$ we see, when we use the bound for mass in (1.32), that (3.13) reduces to

$$
k^{-(p+m-3)} t^{-1} \rho\left(R_{\rho}\left(k^{-1}\left\|\rho u_{0}\right\|_{1}\right)\right) R_{\rho}\left(k^{-1}\left\|\rho u_{0}\right\|_{1}\right)^{p}=\gamma_{0} .
$$

We conclude the proof by invoking Remark 1.4.

\section{Proof of the Sup Estimate in the Case $p+m-3<0$}

We borrow the notation from Sect. 3, assuming also $\theta>3-p-m$ is so large that $r<p$, and that $s>p$ satisfies (2.52): see Remark 2.12. Let us also note explicitly that assumption (1.34), with some elementary algebra, yields

$$
\left(N-\alpha_{1}\right)(p-s)+r\left(p-\alpha_{2}\right)>0 .
$$

which in turn implies immediately $\mathcal{H}(s)>0(\mathcal{H}$ has been defined in Corollary 2.15).

We start again from (3.1); on the right hand side there by means of (2.76), we get

$$
\begin{aligned}
& \int_{M} \rho v_{n+1}^{s} \mathrm{~d} \mu \leq \gamma\left(\int_{M} \rho v_{n+1}^{r} \mathrm{~d} \mu\right)^{\frac{\mathcal{H}(s)}{\mathcal{H}(r)}} \\
& \quad \times W(\Sigma)^{\frac{(s-r)\left(N-\alpha_{1}\right)}{\mathcal{H}(r)}}\left(\int_{M}\left|\nabla v_{n+1}\right|^{p} \mathrm{~d} \mu\right)^{\frac{(s-r)\left(N-\alpha_{1}\right)}{\mathcal{H}(r)}},
\end{aligned}
$$

where $\Sigma$ is then bounded as in (3.4). From now on, the proof proceeds formally unchanged as in Sect. 3, with the remarks below.

We use Hölder inequality to bound the right hand side of (3.1) exactly with the right hand side of (3.5) (with the current values of $s, r$ ). Note that the condition

$$
\frac{(s-r)\left(N-\alpha_{1}\right)}{\mathcal{H}(r)}<1,
$$

required in order to apply Hölder (and, next, Young) inequality, is not automatically satisfied as in the case of Sect. 3; anyway, it is easily seen by a direct elementary calculation that (4.3) is equivalent to (4.1).

Then we apply Young inequality to arrive at (3.8). Here we specifically note that all exponents have the expected sign owing to (4.1), and that in (3.9) $\eta$ is positive by assumption (1.34).

At the end of the proof, in order to define $k$ by means of (3.14), we exploit our assumption that $\psi$ is increasing. 


\section{Proof of the Finite Speed of Propagation}

Here we introduce the notation

$$
\begin{aligned}
& A_{n}=\left\{x \in M \mid R_{n}^{\prime}<d(x)<R_{n}^{\prime \prime}\right\}, \quad 0<\eta, \sigma \leq \frac{1}{4}, \quad R \geq 4 R_{0}, \\
& R_{n}^{\prime}=\frac{R}{2}\left(1-\eta-\sigma+\sigma 2^{-n}\right), \quad R_{n}^{\prime \prime}=\frac{R}{2}\left(1+\eta+\sigma-\sigma 2^{-n}\right) .
\end{aligned}
$$

We also introduce a standard cutoff function $\zeta_{n} \in C_{0}^{1}\left(A_{n+1}\right)$ such that

$$
0 \leq \zeta_{n} \leq 1 ; \quad \zeta_{n}(x)=1, \quad x \in A_{n} ; \quad\left|\nabla \zeta_{n}(x)\right| \leq \gamma 2^{n}(\sigma R)^{-1} .
$$

Then for $\theta>0$ as in Sect. 3 we define

$$
r=\frac{p}{p+m+\theta-2}, \quad s=(1+\theta) r<p, \quad v_{n}=\left(u \zeta_{n}\right)^{\frac{p+m+\theta-2}{p}} .
$$

Note that no $A_{n}$ intersects $\operatorname{supp} u_{0}$. Then from Lemma 2.18 we get

$$
\begin{aligned}
J_{n} & :=\sup _{0<\tau<t} \int_{M} v_{n}^{s} \rho \mathrm{d} \mu+\int_{0}^{t} \int_{M}\left|\nabla v_{n}\right|^{p} \mathrm{~d} \mu \mathrm{d} \tau \\
& \leq \gamma \frac{2^{n p}}{\sigma^{p} R^{p}} \int_{0}^{t} \int_{M} v_{n+1}^{p} \mathrm{~d} \mu \mathrm{d} \tau .
\end{aligned}
$$

Next we apply Corollary 2.9 to $v_{n+1}$ and get with the present choice of $r<p$ (remember that the support of $v_{n+1}$ is contained in an annulus), and for all times,

$$
\begin{aligned}
& \int_{M} v_{n+1}^{p} \mathrm{~d} \mu \leq \gamma \omega(V(R))^{\frac{p N(p-r)}{N(p-r)+r p}}\left(\int_{M} v_{n+1}^{r} \mathrm{~d} \mu\right)^{\frac{p^{2}}{N(p-r)+r p}} \\
& \quad \times\left(\int_{M}\left|\nabla v_{n+1}\right|^{p} \mathrm{~d} \mu\right)^{\frac{N(p-r)}{N(p-r)+r p}} .
\end{aligned}
$$

We integrate in time this estimate, and apply Young inequality, to bound above the right hand side of (5.1) with

$\varepsilon \int_{0}^{t}\left|\nabla v_{n+1}\right|^{p} \mathrm{~d} \mu+\gamma \varepsilon^{-\frac{N(p-r)}{r p}} t\left(\frac{2^{n}}{\sigma R}\right)^{\frac{N(p-r)+r p}{r}} \omega(V(R))^{\frac{N(p-r)}{r}} \sup _{0<\tau<t}\left(\int_{M} v_{n+1}^{r} \mathrm{~d} \mu\right)^{\frac{p}{r}}$.

From (5.1) and (5.3) we infer, on substituting the definition of $r$, the recursive inequality

$$
\begin{aligned}
J_{n} \leq & \varepsilon J_{n+1}+\gamma \varepsilon^{-\frac{N}{p}(p+m+\theta-3)}\left(\frac{2^{n}}{\sigma}\right)^{N(p+m+\theta-3)+p} \\
& \times t V(R)^{-(p+m+\theta-3)} R^{-p} \sup _{0<\tau<t}\left(\int_{A_{\infty}} u \mathrm{~d} \mu\right)^{p+m+\theta-2},
\end{aligned}
$$

where

$$
A_{\infty}=\left\{x \in M \mid \frac{R}{2}(1-\eta-\sigma)<d(x)<\frac{R}{2}(1+\eta+\sigma)\right\} .
$$


A standard iterative argument, relying on a suitable choice of $\varepsilon$, then yields the estimate

$$
\begin{aligned}
& \sup _{0<\tau<t} \int_{A_{0}} \rho u^{1+\theta} \mathrm{d} \mu \leq \gamma \sigma^{-N(p+m+\theta-3)-p} \\
& \quad \times t V(R)^{-(p+m+\theta-3)} R^{-p} \sup _{0<\tau<t}\left(\int_{A_{\infty}} u \mathrm{~d} \mu\right)^{p+m+\theta-2} .
\end{aligned}
$$

Define next a sequence of shrinking annuli

$$
\begin{aligned}
D_{n} & =\left\{x \in M \mid \bar{R}_{n}^{\prime}<d(x)<\bar{R}_{n}^{\prime \prime}\right\}, \\
\bar{R}_{n}^{\prime} & =\frac{R}{2}\left(1-2^{-n-1}\right), \quad \bar{R}_{n}^{\prime \prime}=\frac{R}{2}\left(1+2^{-n-1}\right) .
\end{aligned}
$$

We apply inequality (5.5) with $A_{0}=D_{n+1}, A_{\infty}=D_{n}, \sigma=\eta=2^{-n-2}, n \geq 0$, and obtain, using the fact that $\rho(d(x)) \geq \rho(R)$ in $D_{n}$,

$$
\begin{aligned}
& \sup _{0<\tau<t} \int_{D_{n+1}} \rho u^{1+\theta} \mathrm{d} \mu \leq \gamma\left(2^{n}\right)^{N(p+m+\theta-3)-p} \\
& \quad \times t V(R)^{-(p+m+\theta-3)} R^{-p} \rho(R)^{-(p+m+\theta-2)} \sup _{0<\tau<t}\left(\int_{D_{n}} \rho u \mathrm{~d} \mu\right)^{p+m+\theta-2} .
\end{aligned}
$$

We get from Hölder inequality that

$$
\begin{aligned}
Y_{n}:= & \sup _{0<\tau<t} \int_{D_{n}} \rho u \mathrm{~d} \mu \leq\left(\int_{D_{n}} \rho \mathrm{d} \mu\right)^{\frac{\theta}{1+\theta}} \\
& \times\left(\sup _{0<\tau<t} \int_{D_{n}} \rho u^{1+\theta} \mathrm{d} \mu\right)^{\frac{1}{1+\theta}} \\
\leq & \gamma(V(R) \rho(R))^{\frac{\theta}{1+\theta}}\left(\sup _{0<\tau<t} \int_{D_{n}} \rho u^{1+\theta} \mathrm{d} \mu\right)^{\frac{1}{1+\theta}},
\end{aligned}
$$

where we also took into account (1.36).

From (5.6) and (5.7) we infer at once

$$
Y_{n+1} \leq \gamma b^{n}\left(\frac{t}{V(R)^{p+m-3} R^{p} \rho(R)^{p+m-2}}\right)^{\frac{1}{1+\theta}} Y_{n}^{1+\frac{p+m-3}{1+\theta}},
$$

where $b$ is a suitable power of 2 . It follows from [26, Lemma 5.6, Chapt. II] that $Y_{n} \rightarrow 0$ as $n \rightarrow \infty$ if $R$ is chosen so that

$$
\frac{t}{V(R)^{p+m-3} R^{p} \rho(R)^{p+m-2}}\left(\int_{M} \rho u_{0} \mathrm{~d} \mu\right)^{p+m-3} \leq \gamma_{0}
$$

for a suitable constant $\gamma_{0}>0$ depending on the parameters of the problem. We also use here the bound in (1.32).

Finally, note that according to the definition of $Y_{n}$ we proved that $u(x, t)=0$ for $x \in M \backslash B_{R}$ if $R$ satisfies (5.9) and of course the condition supp $u_{0} \subset B_{R / 4}$ stated at the beginning of the proof. We have thus proved the sought after result. 


\section{Proof of the Universal Bound}

We need the following

Lemma 6.1. Assume that the assumptions of Lemma 2.6, (1.23), (1.24) hold true, and that for $\alpha>0, \beta \in(0, N)$ we have for a suitable $c>0$

$$
\rho(\tau) \leq c^{-1} \tau^{-\alpha}, \quad \tau>1
$$

and

$$
V(\tau) \geq c \tau^{\beta}, \quad \tau>1
$$

In addition we require that one of the following holds:

$$
\begin{aligned}
& p<\beta, \quad \alpha \geq \beta, \quad 0<r<p^{*}, \\
& p<\beta, \quad p \frac{N-\beta}{N-p}<\alpha<\beta, \quad p \frac{\beta-\alpha}{\beta-p}<r<p^{*}, \\
& p=\beta, \quad \alpha>\beta, \quad 0<r<p^{*}, \\
& p>\beta, \quad 0<r<\min \left(p^{*}, p \frac{\alpha-\beta}{p-\beta}\right) .
\end{aligned}
$$

Then

$$
\int_{M} \rho|u|^{r} \mathrm{~d} \mu \leq \gamma\left(\int_{M}|\nabla u|^{p} \mathrm{~d} \mu\right)^{\frac{r}{p}}
$$

Proof. From Hölder inequality and Lemma 2.6 we infer

$$
\begin{aligned}
& \int_{M} \rho|u|^{r} \mathrm{~d} \mu \leq\left(\int_{M}|u|^{p^{*}} \omega(V(d(x)))^{-p^{*}} \mathrm{~d} \mu\right)^{\frac{r}{p^{*}}} J^{1-\frac{r}{p^{*}}} \\
& \quad \leq \gamma\left(\int_{M}|\nabla u|^{p} \mathrm{~d} \mu\right)^{\frac{r}{p}} J^{1-\frac{r}{p^{*}}}
\end{aligned}
$$

where

$$
\begin{aligned}
J & :=\int_{M} \rho(d(x))^{\frac{p^{*}}{p^{*}-r}} \omega(V(d(x)))^{\frac{r p^{*}}{p^{*}-r}} \mathrm{~d} \mu \\
& \leq \gamma+\int_{M \backslash B_{1}} \rho(d(x))^{\frac{p^{*}}{p^{*}-r}} \omega(V(d(x)))^{\frac{r p^{*}}{p^{*}-r}} \mathrm{~d} \mu .
\end{aligned}
$$

We majorize the last integral as

$$
\begin{aligned}
& \int_{1}^{+\infty} \rho(\tau)^{\frac{p^{*}}{p^{*}-r}} \omega(V(\tau))^{\frac{r p^{*}}{p^{*}-r}} \frac{\mathrm{d} V}{\mathrm{~d} \tau}(\tau) \mathrm{d} \tau \\
& \quad \leq \gamma \int_{1}^{+\infty} \rho(\tau)^{\frac{p^{*}}{p^{*}-r}} \tau^{\frac{r p^{*}}{p^{*}-r}-1} V(\tau)^{1-\frac{r p^{*}}{N\left(p^{*}-r\right)}} \mathrm{d} \tau,
\end{aligned}
$$

where we have used the definition of $\omega$ and (1.23) and (1.24). Finally, the right hand side of (6.6) is bounded by

$$
\int_{1}^{+\infty} \tau^{\frac{p^{*}(r-\alpha)}{p^{*}-r}-1+\beta-\frac{\beta r p^{*}}{N\left(p^{*}-r\right)}} \mathrm{d} \tau,
$$

according to our assumptions on $V$ and $\rho$. The last integral converges in the cases given in the statement, as a direct inspection shows. 
Proof of Theorem. We use the notation introduced in Sect. 3, with the exception that while we still denote $s=p(1+\theta) /(p+m+\theta-2)<p, \theta>0$, we choose $p<r<p^{*}$. We may apply Lemma 6.1 , since (6.2) with $\beta=p$ follows under our assumptions from (2.4), so that $p<r<p^{*}$ is in the admissible range for Lemma 6.1. Then we have at every time level, since $s<p<r$,

$$
\begin{aligned}
& \int_{M} \rho v_{n+1}^{s} \mathrm{~d} \mu \leq\left(\int_{M} \rho v_{n+1}^{r} \mathrm{~d} \mu\right)^{\frac{s}{r}} \mu_{\rho}\left(\left\{u>k_{n+1}\right\}\right)^{1-\frac{s}{r}} \\
& \quad \leq \gamma\left(\int_{M}\left|\nabla v_{n+1}\right|^{p} \mathrm{~d} \mu\right)^{\frac{s}{p}} \mu_{\rho}\left(\left\{u>k_{\infty}\right\}\right)^{1-\frac{s}{r}} .
\end{aligned}
$$

Then we apply to (3.1) the estimate (6.8) and Young inequality; with the notation (3.7), we arrive at

$$
\begin{aligned}
I_{n} \leq & \varepsilon \int_{\tau_{n+1}}^{t} \int_{M}\left|\nabla v_{n+1}\right|^{p} \mathrm{~d} \mu \mathrm{d} \tau \\
& +\gamma \varepsilon^{-\frac{s}{p-s}} \frac{2^{\frac{n \ell p}{p-s}}}{\sigma^{\frac{\ell p}{p-s}}} t^{-\frac{s}{p-s}} \sup _{\tau_{\infty}<\tau<t} \mu_{\rho}\left(\left\{u(\tau)>k_{\infty}\right\}\right)^{\frac{p(r-s)}{r(p-s)}} .
\end{aligned}
$$

Then an iterative argument essentially identical to the one employed in (3.6)-(3.8) leads us, for a suitable choice of $\varepsilon>0$, to

$$
\begin{aligned}
& \sup _{\tau_{0}<\tau<t} \int_{M} \rho\left(u(\tau)-k_{0}\right)_{+}^{1+\theta} \mathrm{d} \mu \\
& \quad \leq \gamma \sigma^{-\frac{\ell(p+m+\theta-2)}{p+m-3}} t^{-\frac{1+\theta}{p+m-3}} \sup _{\tau_{\infty}<\tau<t} \mu_{\rho}\left(\left\{u(\tau)>k_{\infty}\right\}\right)^{1+\frac{(r-p)(1+\theta)}{r(p+m-3)}} .
\end{aligned}
$$

Note that the last exponent is greater than 1 owing to our choice $r>p$.

We proceed as in Sect. 3, introducing $h_{j}, y_{j}$ and $Y_{i}=y_{2 i}$ as there. When we take into account that

$$
\mu_{\rho}\left(\left\{u(\tau)>h_{j}\right\}\right) \leq 2^{j+2} k^{-1} y_{j-1},
$$

we get (remember that we set $k_{\infty}=h_{j}, k_{0}=h_{j+1}$ )

$$
Y_{i+1} \leq \gamma 4 \frac{\left(\frac{\ell(p+m+\theta-2)}{p+m-3}+\theta+1\right) i}{k^{-(\theta+1)}\left(1+\frac{r-p}{r(p+m-3)}\right)} t^{-\frac{1+\theta}{p+m-3}} Y_{i}^{1+\frac{(r-p)(1+\theta)}{r(p+m-3)}},
$$

for $i \geq 0$. It follows from [26, Lemma 5.6, Chapt. II] that $Y_{i} \rightarrow 0$ as $i \rightarrow+\infty$, i.e., $u(t) \leq k$, if

$$
k^{-1-\frac{r-p}{r(p+m-3)}} t^{-\frac{1}{p+m-3}} Y_{0}^{\frac{r-p}{r(p+m-3)}} \leq \gamma_{0}
$$

for a suitable constant $\gamma_{0}>0$ depending on the parameters. We bound the integral appearing in $Y_{0}$ (at each time level) as

$$
\begin{aligned}
& \int_{M} \rho\left(u-\frac{3}{4} k\right)_{+} \mathrm{d} \mu \leq\left(\int_{M} \rho\left(u-\frac{3}{4} k\right)_{+}^{q+1} \mathrm{~d} \mu\right)^{\frac{1}{q+1}} \mu_{\rho}\left(\left\{u>\frac{3}{4} k\right\}\right)^{\frac{q}{q+1}} \\
& \quad \leq\left(\int_{M} \rho\left(u-\frac{3}{4} k\right)_{+}^{q+1} \mathrm{~d} \mu\right)^{\frac{1}{q+1}}\left(\left(4 k^{-1}\right)^{q+1} \int_{M} \rho\left(u-\frac{k}{2}\right)_{+}^{q+1} \mathrm{~d} \mu\right)^{\frac{q}{q+1}} \\
& \leq\left(4 k^{-1}\right)^{q} \int_{M} \rho u^{q+1} \mathrm{~d} \mu,
\end{aligned}
$$


for $q>0$. It follows then from (6.12) (with an equality) that

$$
\|u(t)\|_{\infty} \leq \gamma t^{-\frac{r}{\mathcal{K}}} \sup _{t / 4<\tau<t} E_{q+1}(\tau)^{\frac{r-p}{\mathcal{K}}}, \quad t>0
$$

for $E_{q+1}$ defined as in $(2.59)$, and

$$
\mathcal{K}=r(p+m-3)+(r-p)(q+1) .
$$

We are left with the task of estimating $E_{q+1}(\tau)$; this will be accomplished by appealing again to Lemma 6.1 , where we select

$$
0<r^{\prime}:=\frac{p(1+q)}{p+m+q-2}<p<p^{*} .
$$

We obtain from the differential equation (1.1), for $w=u^{(p+m+q-2) / p}$ the equality in

$$
\frac{1}{q+1} \frac{\mathrm{d} E_{q+1}}{\mathrm{~d} t}=-\left(\frac{p}{p+m+\theta-2}\right)^{p} \int_{M}|\nabla w|^{p} \mathrm{~d} \mu \leq-\gamma E_{q+1}(t)^{\frac{p+m+q-2}{1+q}},
$$

where the inequality follows from an application of (6.3) with $r$ replaced with the $r^{\prime}$ given in (6.14). On integrating (6.15) we get

$$
E_{q+1}(t) \leq \gamma t^{-\frac{1+q}{p+m-3}}, \quad t>0 ;
$$

actually we integrate over $\left(t_{0}, t\right)$ and then let $t_{0} \rightarrow 0+$ in order to circumvent possible problems with the local summability of the initial data. Finally we substitute (6.16) in (6.13) to prove the claim of the Theorem.

\section{Interface Blow Up}

Let us reason by contradiction, and assume that the support of $u(t)$ stays bounded for all times.

Then we get for all times and for a fixed $\theta>0$, by means of Hölder and Hardy inequality

$$
\begin{aligned}
& \int_{M} \rho u \mathrm{~d} \mu \leq\left(\int_{M} d(x)^{-p} u^{p+m+\theta-2} \mathrm{~d} \mu\right)^{\frac{1}{p+m+\theta-2}} I(\theta)^{\frac{p+m+\theta-3}{p+m+\theta-2}} \\
& \quad \leq \gamma\left(\int_{M}\left|\nabla u^{\frac{p+m+\theta-2}{p}}\right|^{p} \mathrm{~d} \mu\right)^{\frac{1}{p+m+\theta-2}} I(\theta)^{\frac{p+m+\theta-3}{p+m+\theta-2}},
\end{aligned}
$$

where, when we recall $(1.23)$

$$
\begin{aligned}
I(\theta) & =\int_{M}\left(d(x)^{p} \rho(x)^{p+m+\theta-2}\right)^{\frac{1}{p+m+\theta-3}} \mathrm{~d} \mu \\
& \leq N \int_{0}^{+\infty}\left(\tau^{p} \rho(\tau)^{p+m+\theta-2}\right)^{\frac{1}{p+m+\theta-3}} \frac{V(\tau)}{\tau} \mathrm{d} \tau \\
& =N \int_{0}^{+\infty}\left(\tau^{p} \rho(\tau)\right)^{-\frac{\theta}{(p+m-3)(p+m+\theta-3)}} \psi(\tau)^{\frac{1}{p+m-3}} \frac{\mathrm{d} \tau}{\tau}<+\infty,
\end{aligned}
$$

by assumption (1.40), if $\theta$ is chosen suitably small. 
By means of calculation in all similar we get

$$
\begin{aligned}
& \int_{M} \rho u^{1+\theta} \mathrm{d} \mu \leq\left(\int_{M} d(x)^{-p} u^{p+m+\theta-2} \mathrm{~d} \mu\right)^{\frac{1+\theta}{p+m+\theta-2}} J(\theta)^{\frac{p+m-3}{p+m+\theta-2}} \\
& \quad \leq \gamma\left(\int_{M}\left|\nabla u^{\frac{p+m+\theta-2}{p}}\right|^{p} \mathrm{~d} \mu\right)^{\frac{1+\theta}{p+m+\theta-2}} J(\theta)^{\frac{p+m-3}{p+m+\theta-2}}
\end{aligned}
$$

where when we recall $(1.23)$

$$
\begin{aligned}
J(\theta) & =\int_{M}\left(d(x)^{p(1+\theta)} \rho(x)^{p+m+\theta-2}\right)^{\frac{1}{p+m-3}} \mathrm{~d} \mu \\
& \leq N \int_{0}^{+\infty}\left(\tau^{p(1+\theta)} \rho(\tau)^{p+m+\theta-2}\right)^{\frac{1}{p+m-3}} \frac{V(\tau)}{\tau} \mathrm{d} \tau \\
& =N \int_{0}^{+\infty}\left(\tau^{p} \rho(\tau)\right)^{\frac{\theta}{p+m-3}} \psi(\tau)^{\frac{1}{p+m-3}} \frac{\mathrm{d} \tau}{\tau}<+\infty,
\end{aligned}
$$

again by assumption (1.40), for a suitable choice of $\theta$.

On using (7.2) and the differential equation (1.1), we obtain

$$
\begin{aligned}
& \frac{1}{1+\theta} \frac{\mathrm{d}}{\mathrm{d} t} \int_{M} \rho u(t)^{1+\theta} \mathrm{d} \mu=-\left(\frac{p}{p+m+\theta-2}\right)^{p} \int_{M}\left|\nabla u^{\frac{p+m+\theta-2}{p}}\right|^{p} \mathrm{~d} \mu \\
& \quad \leq-\gamma\left(\int_{M} \rho u(t)^{1+\theta} \mathrm{d} \mu\right)^{\frac{p+m+\theta-2}{1+\theta}},
\end{aligned}
$$

whence

$$
\int_{M} \rho u(t)^{1+\theta} \mathrm{d} \mu \leq \gamma t^{-\frac{1+\theta}{p+m-3}}, \quad t>0 .
$$

However, (7.1) and Hölder inequality yield

$$
\int_{t}^{t+1} \int_{M} \rho u \mathrm{~d} \mu \mathrm{d} \tau \leq\left(\int_{t}^{t+1} \int_{M}\left|\nabla u^{\frac{p+m+\theta-2}{p}}\right|^{p} \mathrm{~d} \mu \mathrm{d} \tau\right)^{\frac{1}{p+m+\theta-2}} .
$$

Again integrating the equality in (7.3), we get

$$
\int_{t}^{t+1} \int_{M}\left|\nabla u^{\frac{p+m+\theta-2}{p}}\right|^{p} \mathrm{~d} \mu \mathrm{d} \tau \leq \gamma \int_{M} \rho u(t)^{1+\theta} \mathrm{d} \mu,
$$

and finally on combining (7.4) with (7.6) we obtain

$$
\int_{M} \rho u_{0} \mathrm{~d} \mu=\int_{t}^{t+1} \int_{M} \rho u(t) \mathrm{d} \mu \mathrm{d} \tau \leq \gamma t^{-\frac{1+\theta}{(p+m+3)(p+m+\theta-2)}},
$$

at least for all the times $t$ such that the compactness of the support of $u(\tau)$ holds true over $(0, t+1)$. Indeed it is known, as we recalled in Remark 1.5, that such a property implies conservation of mass. But, (7.7) clearly is inconsistent as $t \rightarrow+\infty$, completing our argument.

Funding Open access funding provided by Universitá degli Studi di Roma La Sapienza within the CRUI-CARE Agreement. 
Open Access. This article is licensed under a Creative Commons Attribution 4.0 International License, which permits use, sharing, adaptation, distribution and reproduction in any medium or format, as long as you give appropriate credit to the original author(s) and the source, provide a link to the Creative Commons licence, and indicate if changes were made. The images or other third party material in this article are included in the article's Creative Commons licence, unless indicated otherwise in a credit line to the material. If material is not included in the article's Creative Commons licence and your intended use is not permitted by statutory regulation or exceeds the permitted use, you will need to obtain permission directly from the copyright holder. To view a copy of this licence, visit http://creativecommons.org/licenses/by/4.0/.

\section{References}

[1] Andreucci, D., Cirmi, R., Leonardi, S., Tedeev, A.F.: Large time behavior of solutions to the Neumann problem for a quasilinear second order degenerate parabolic equation in domains with noncompact boundary. J. Differ. Equ. 174, 253-288 (2001)

[2] Andreucci, D., Tedeev, A.: Extinction in a finite time for parabolic equations of fast diffusion type on manifolds. In: Kusraev, A.G., Totieva, Z.D. (eds.) Operator Theory and Differential Equations. Trends in Mathematics, pp. 1-6. Birkhäuser, Basel (2021)

[3] Andreucci, D., Tedeev, A.F.: Optimal bounds and blow up phenomena for parabolic problems in narrowing domains. Proc. R. Soc. Edinb. 128A, 1163-1180 (1998)

[4] Andreucci, D., Tedeev, A.F.: A Fujita type result for a degenerate Neumann problem in domains with non compact boundary. J. Math. Anal. Appl. 231, 543-567 (1999)

[5] Andreucci, D., Tedeev, A.F.: Sharp estimates and finite speed of propagation for a Neumann problem in domains narrowing at infinity. Adv. Differ. Equ. 5, 833-860 (2000)

[6] Andreucci, D., Tedeev, A.F.: Universal bounds at the blow-up time for nonlinear parabolic equations. Adv. Differ. Equ. 10, 89-120 (2005)

[7] Andreucci, D., Tedeev, A.F.: Optimal decay rate for degenerate parabolic equations on noncompact manifolds. Methods Appl. Anal. 22(4), 359-376 (2015)

[8] Andreucci, D., Tedeev, A.F.: Large time behavior for the porous medium equation with convection. Meccanica 52(13), 3255-3260 (2017)

[9] Aubin, T.: Some Nonlinear Problems in Riemannian Geometry. Springer Monographs in Mathematics, Springer, Berlin (1998)

[10] Bernis, F.: Existence results for doubly nonlinear higher order parabolic equations on unbounded domains. Mathematische Annalen 279, 373-394 (1988)

[11] Chavel, I.: Isoperimetric Inequalities: Differential Geometric and Analytic Perspectives. Cambridge Tracts in Mathematics, vol. 145. Cambridge University Press, Cambridge (2001)

[12] D'Ambrosio, L., Dipierro, S.: Hardy inequalities on Riemannian manifolds and applications. Ann. Inst. H. Poincaré Anal. Non Linéaire 31(3), 449-475 (2014)

[13] DiBenedetto, E.: Degenerate Parabolic Equations. Springer, New York (1993)

[14] Düzgün, F.G., Mosconi, S., Vespri, V.: Anisotropic Sobolev embeddings and the speed of propagation for parabolic equations. J. Evol. Equ. 19(3), 845-882 (2019)

[15] Dzagoeva, L.F., Tedeev, A.F.: Asymptotic behavior of the solution of doubly degenerate parabolic equations with inhomogeneous density (submitted)

[16] Eidus, D., Kamin, S.: The filtration equation in a class of functions decreasing at infinity. Proc. Am. Math. Soc. 120(3), 825-830 (1994) 
[17] Grigoryan, A.: Heat kernels on weighted manifolds and applications. In: The Ubiquitous Heat Kernel. Contemporary Mathematics, vol. 398, pp. 93-191. American Mathematical Society, Providence (2006)

[18] Grigoryan, A., Saloff-Coste, L.: Surgery of the Faber-Krahn inequality and applications to heat kernel bounds. Nonlinear Anal. 131, 243-272 (2016)

[19] Grillo, G., Muratori, M.: Sharp short and long time $L^{\infty}$ bounds for solutions to porous media equations with homogeneous Neumann boundary conditions. J. Differ. Equ. 254(5), 2261-2288 (2013)

[20] Grillo, G., Muratori, M.: Smoothing effects for the porous medium equation on CartanHadamard manifolds. Nonlinear Anal. 131, 346-362 (2016)

[21] Grillo, G., Muratori, M., Porzio, M.M.: Porous media equations with two weights: smoothing and decay properties of energy solutions via Poincaré inequalities. Discret. Contin. Dyn. Syst. 33(8), 3599-3640 (2013)

[22] Iagar, R.G., Sánchez, A.: Asymptotic behavior for the heat equation in nonhomogeneous media with critical density. Nonlinear Anal. 89, 24-35 (2013)

[23] Kamin, S., Kersner, R.: Disappearance of interfaces in finite time. Meccanica 28(2), 117-120 (1993)

[24] Kamin, S., Reyes, G., Vázquez, J.L.: Long time behavior for the inhomogeneous PME in a medium with rapidly decaying density. Discrete Contin. Dyn. Syst. 26(2), 521-549 (2010)

[25] Kamin, S., Rosenau, P.: Propagation of thermal waves in an inhomogeneous medium. Commun. Pure Appl. Math. 34(6), 831-852 (1981)

[26] Ladyzhenskaja, O.A., Solonnikov, V.A., Uralceva, N.N.: Linear and Quasilinear Equations of Parabolic Type. Translations of Mathematical Monographs, vol. 23. American Mathematical Society, Providence (1968)

[27] Martynenko, A.V., Tedeev, A.F.: The Cauchy problem for a quasilinear parabolic equation with a source and nonhomogeneous density. Zh. Vychisl. Mat. Mat. Fiz. 47(2), 245-255 (2007)

[28] Martynenko, A.V., Tedeev, A.F.: On the behavior of solutions of the Cauchy problem for a degenerate parabolic equation with nonhomogeneous density and a source. Zh. Vychisl. Mat. Mat. Fiz. 48(7), 1214-1229 (2008)

[29] Mazya, V.G.: Sobolev Spaces. Springer Series in Soviet Mathematics, Springer, Berlin (1985)

[30] Minerbe, V.: Weighted Sobolev inequalities and Ricci flat manifolds. Geom. Funct. Anal. 18(5), 1696-1749 (2009)

[31] Nieto, S., Reyes, G.: Asymptotic behavior of the solutions of the inhomogeneous porous medium equation with critical vanishing density. Commun. Pure Appl. Anal. 12(2), 1123-1139 (2013)

[32] Punzo, F.: On support of solutions to singular nonlinear parabolic equations in bounded domains. Interfaces Free Bound. 13(3), 397-410 (2011)

[33] Punzo, F.: Support properties of solutions to nonlinear parabolic equations with variable density in the hyperbolic space. Discret. Contin. Dyn. Syst. Ser. S 5(3), 657-670 (2012)

[34] Punzo, F.: Uniqueness and non-uniqueness of solutions to quasilinear parabolic equations with a singular coefficient on weighted Riemannian manifolds. Asymptot. Anal. 79(3-4), 273-301 (2012) 
[35] Punzo, F.: Uniqueness and support properties of solutions to singular quasilinear parabolic equations on surfaces of revolution. Ann. Mat. Pura Appl. (4) 191(2), 311-338 (2012)

[36] Reyes, G., Vázquez, J.L.: Long time behavior for the inhomogeneous PME in a medium with slowly decaying density. Commun. Pure Appl. Anal. 8(2), 493-508 (2009)

[37] Ritoré, M., Vernadakis, E.: Large isoperimetric regions in the product of a compact manifold with Euclidean space. Adv. Math. 306, 958-972 (2017)

[38] Rosenau, P., Kamin, S.: Nonlinear diffusion in a finite mass medium. Commun. Pure Appl. Math. 35(1), 113-127 (1982)

[39] Ruiz, J.M., Juárez, A.V.: Isoperimetric estimates in low dimensional Riemannian products. Ann. Global Anal. Geom. 59(4), 417-434 (2021). https://doi.org/10.1007/ s10455-021-09757-6

[40] Talenti, G.: Elliptic equations and rearrangements. Annali Scuola Normale Superiore di Pisa 3, 697-718 (1976)

[41] Tedeev, A.F.: Qualitative properties of solutions of Neumann problem for quasilinear higher order parabolic equations. Ukr. Math. J. 44, 1571-1579 (1993)

[42] Tedeev, A.F.: The interface blow-up phenomenon and local estimates for doubly degenerate parabolic equations. Appl. Anal. 86(6), 755-782 (2007)

[43] Varopoulos, N.T.: Hardy-Littlewood theory for semigroups. J. Funct. Anal. 63(2), 240$260(1985)$

[44] Vázquez, J.L.: Fundamental solution and long time behavior of the porous medium equation in hyperbolic space. J. Math. Pures Appl. (9) 104(3), 454-484 (2015)

Daniele Andreucci

Department of Basic and Applied Sciences for Engineering

Sapienza University of Rome

via A. Scarpa 16

00161 Rome

Italy

e-mail: daniele.andreucci@sbai.uniroma1.it

Anatoli F. Tedeev

South Mathematical Institute of VSC RAS

Vladikavkaz

Russian Federation

and

North-Caucasus Center for Mathematical Research

Vladikavkaz

Russia

e-mail, a_tedeev@yahoo.com:

Received: April 29, 2021.

Accepted: August 18, 2021. 